
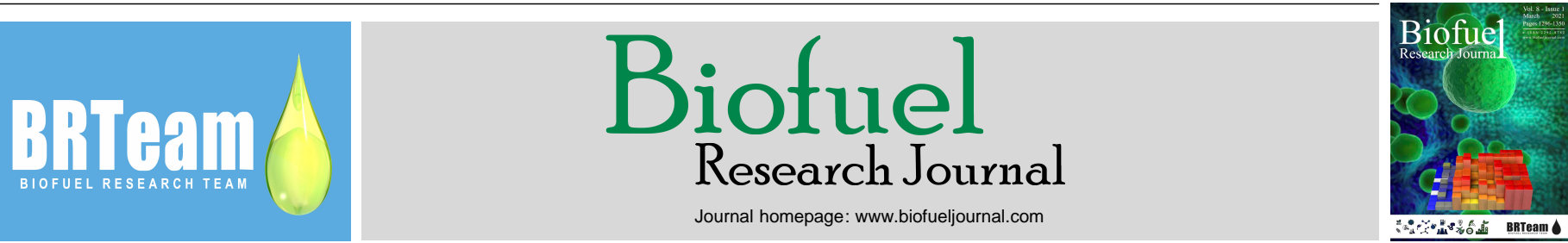

Original Research Paper

\title{
Systematic metabolome profiling and multi-omics analysis of the nitrogen-limited non-model oleaginous algae for biorefining
}

Vineeta Rai ${ }^{1,2,}$, Sandip Kumar Patel ${ }^{1,3, \$}$, Muthusivaramapandian Muthuraj ${ }^{4,5}$, Mayuri N. Gandhi ${ }^{6}$, Debasish Das ${ }^{4,7}$, Sanjeeva Srivastava $a^{1,7, *}$

${ }^{I}$ Department of Biosciences and Bioengineering, Wadhwani Research Center for Biosciences and Bioengineering, Indian Institute of Technology Bombay,

Powai, Mumbai 400076, India.

${ }^{2}$ Department of Entomology \& Plant Pathology, North Carolina State University, Raleigh, NC, 27695, USA.

${ }^{3}$ Buck Institute for Research on Aging, Novato, CA, 94945, USA.

${ }^{4}$ Department of Biosciences and Bioengineering, Centre for Energy, Indian Institute of Technology Guwahati, Assam 781039, India.

${ }^{5}$ Department of Bioengineering, National Institute of Technology Agartala, Tripura 799046, India.

${ }^{6}$ Centre for Research in Nanotechnology \& Science, Indian Institute of Technology Bombay, Powai, Mumbai 400076, India.

${ }^{7}$ DBT PAN IIT Centre for Bioenergy, Indian Institute of Technology Bombay, Powai, Mumbai 400076, India.

\section{HIGHLIGHTS}

$>$ 'Algomics' of non-model nitrogen-limited microalgae to realize metabolic tug-of-war for biomass and TAG assimilation.

$>$ MS/MS annotation reveals accumulation of phytochemicals: TAGs, PUFAs, phytosterols, and terpenoids.

$>4 \times$ and $6 \times$ increase of two different PUFAs: 9(S)HpOTrE and dihomo-gamma-linolenic acid, respectively, during nitrogen-limitation.

$>$ Severity modulation of nitrogen-limitation results in varied TAG species.

$>$ Biorefining TAG and PUFA for economic

feasibility in large-scale biodiesel production.

\section{GRAPHICAL ABSTRACT}

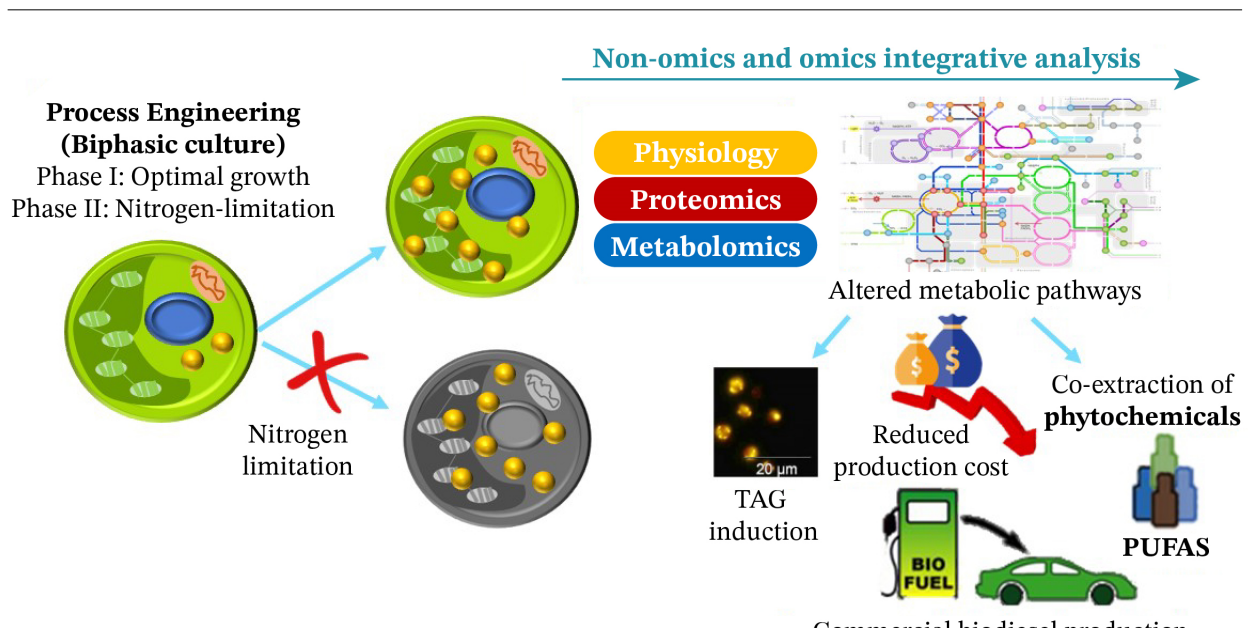

Commercial biodiesel production

* Corresponding author at: Tel.: +91-22-2576-7779
E-mail address: sanjeeva@ iitb.ac.in

\#: These authors contributed equally.

Please cite this article as: Rai V., Patel S.K., Muthuraj M., Gandhi M.N., Das D., Srivastava S. Systematic metabolome profiling and multi-omics analysis of the nitrogen-limited non-model oleaginous algae for biorefining. Biofuel Research Journal 29 (2021) 1330-1341. DOI: 10.18331/BRJ2021.8.1.4 


\section{ARTICLE INFO}

\section{Article history:}

Received 10 November 2020

Received in revised form 26 December 2020

Accepted 4 February 2021

Available online 1 March 2021

\section{Keywords:}

Chlorella sp. FC2 IITG

Metabolomic profiling

Multi-omics analysis

Nitrogen-limitation

Time-course study

Biodiesel

\begin{abstract}
Oleaginous microalga Chlorella is a promising microbial cell factory for producing lipids, transesterifiable to biodiesel, and phytochemical, high-value molecules (HVMs). To better understand the stress-induced oleaginous mechanism of Chlorella sp. FC2 IITG that closely matches with Chlorella sorokiniana based on 18s rRNA gene sequence, we performed 'Algomics' by systematically integrating metabolomics and proteomics data in response to time-resolved nitrogen-limitation: $40 \mathrm{~h}$ (mild), $88 \mathrm{~h}$ (moderate), and $120 \mathrm{~h}$ (severe). Ten HVMs belonging to four biological classes: triacylglycerol (TAG), polyunsaturated fatty acids (PUFA), phytosterols, and terpenoids were annotated using untargeted metabolomics and MS/MS fragmentation pattern match to the spectral library. In particular, the study evidenced $4 \times$ and $6 \times$ increased accumulation of two different PUFA: 9(S)HpOTrE and dihomo-gamma-linolenic acid, respectively, in nitrogen-limitation conditions. Co-extraction of TAG and PUFA could lower the biodiesel production cost for feasible commercialization. The investigation found a maximum accumulation of TAG 59:10 at $40 \mathrm{~h}$, while that for TAG 54:4 was recorded at $88 \mathrm{~h}$, which suggests different TAG species could be induced by regulating nitrogen-limitation severity. Elevated $\beta$-oxidation, glycolysis, and tricarboxylic acid (TCA) cycle identified in proteomics analysis could provide the substrates, phosphoenolpyruvate, pyruvate, and acetyl CoA, for different phytochemical accumulation in response to nitrogen limitation. The multiomics data unraveled a metabolic tug-of-war ongoing between biomass and storage lipid (TAG) accumulation during nitrogen-limitation, which involved multiple processes including hindered $\mathrm{CO}_{2}$ fixation, the supply of energy, reductants, and carbon reallocation from proteins and membrane lipids. These findings provide distinct oleaginous mechanisms in non-model microalgae, Chlorella sp. FC2 IITG, and engineerable targets for microalgal trait improvements.
\end{abstract}

\section{Contents}

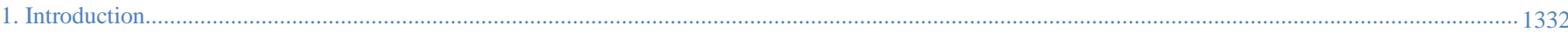

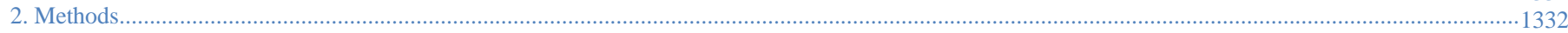

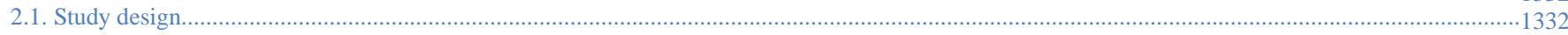

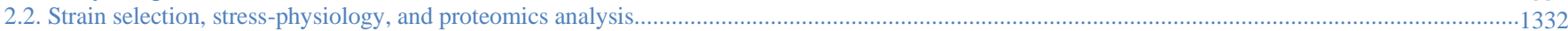

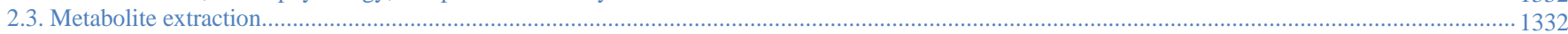

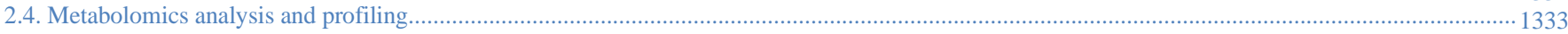

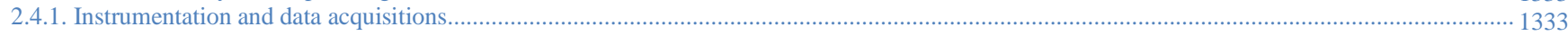

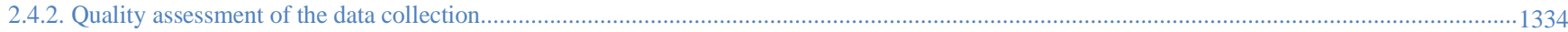

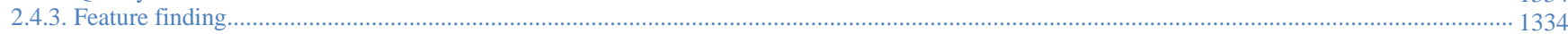

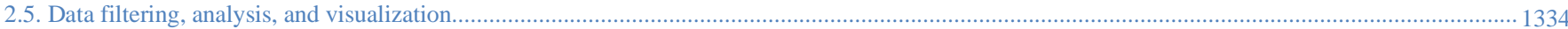

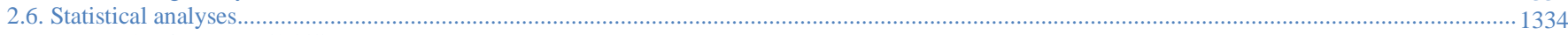

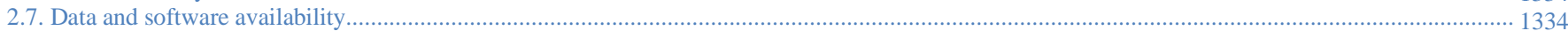

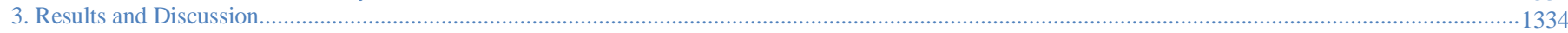

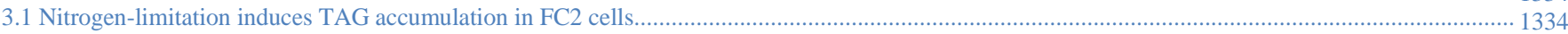

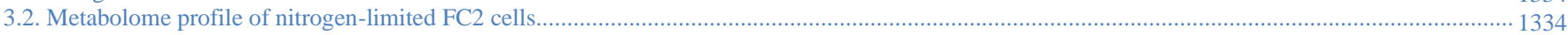

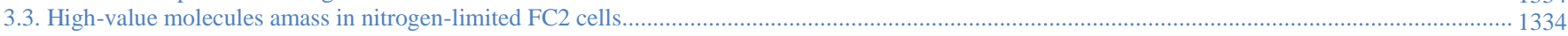

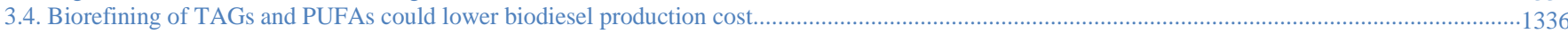

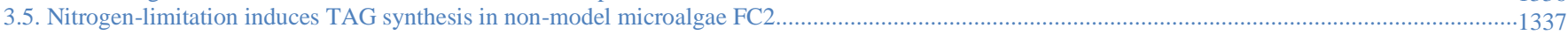

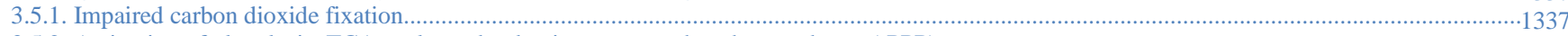

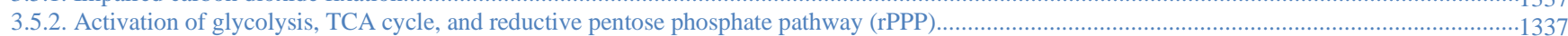

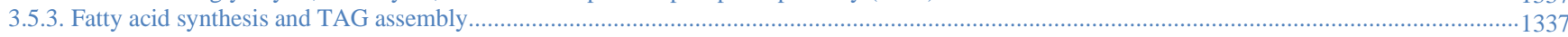

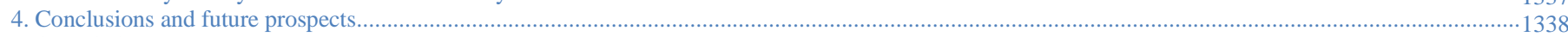

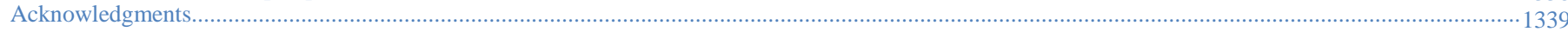

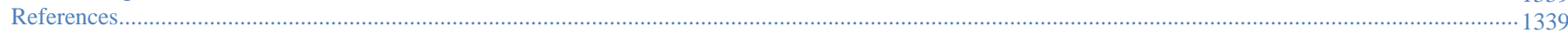

$\begin{array}{llll}\text { Abbreviations } & & & \\ \text { ACCase } & \text { Acetyl-CoA carboxylase } & \text { LC-MS } & \text { Liquid chromatography-mass spectrometry } \\ \text { AGPase } & \text { ADP-gluco-pyro-phosphorylase } & \text { PEP } & \text { Phosphoenolpyruvate } \\ \text { ALDO } & \text { Fructose-1, 6-bisphosphate aldolase } & \text { PGK } & \text { Phosphoglycerate kinase } \\ \text { CV } & \text { Coefficient of variance } & \text { PK } & \text { Pyruvate kinase } \\ \text { CID } & \text { Collision-induced dissociation } & \text { PLS-DA } & \text { Partial least squares-discriminant analysis } \\ \text { DCW } & \text { Dry cell weight } & \text { PRK } & \text { Phosphoribulokinase } \\ \text { DGLA } & \text { Dihomo-gamma-linolenic acid } & \text { PUFA } & \text { Polyunsaturated fatty acid } \\ \text { ENL } & \text { Enolase } & \text { Q-TOF } & \text { Quadrupole time-of-flight } \\ \text { ENR } & \text { Enoyl-ACP reductase } & \text { RPI } & \text { Ribose-5-phosphate isomerase } \\ \text { ESI } & \text { Electrospray ionization } & \text { rPPP } & \text { Reductive pentose phosphate pathway } \\ \text { FAS } & \text { Fatty acid synthase } & \text { RT } & \text { Retention time } \\ \text { FC2 } & \text { Chlorella } \text { sp. FC2 IITG } & \text { SBP } & \text { Sedoheptulose-1,7-bisphosphatase } \\ \text { HAD } & \text { Hydroxy acyl-ACP dehydrogenase } & \text { TAG } & \text { Triacylglycerol } \\ \text { HPLC } & \text { Liquid performance liquid chromatography } & \text { TPI } & \text { Triosephosphate isomerase } \\ \text { HVMs } & \text { High-value molecules } & \text { TCA } & \text { Tricarboxylic acid }\end{array}$




\section{Introduction}

The global energy demand increased by $2.3 \%$ in 2018 (highest since 2010) and is projected to grow by $\sim 50 \%$ between 2018 and 2050 (US Energy Information Administration, 2018). Sustained fossil fuel combustion emits greenhouse gases that contribute significantly to global warming and climatechanges. The global temperature has already risen by $1{ }^{\circ} \mathrm{C}$, and the global temperature is likely to break the $1.5{ }^{\circ} \mathrm{C}$ threshold as early as 2030 if greenhouse gas emissions continue at the current rate. To keep the rising temperatures in check, we need to shift the trajectory of greenhouse gas emissions by 2050 and switch to greener fuels like microalgae-based thirdgeneration biofuels. Microalgae can be used to produce a variety of biofuels, including bioethanol and biodiesel (Colin et al., 2011). However, bioethanol is low in energy density and incompatible with the existing fuel infrastructure, hence encouraging a shift in research focus from algae-based bioethanol to biodiesel production. Biodiesel is an ideal candidate for various reasons: $20 \%$ biodiesel (B20) or lower is suited for current diesel engine models with reliability and durability, superior lubrication properties, non-toxic, biodegradable, and produces lower levels of most air pollutants than petroleumbased diesel fuel. Biodiesel consumption/demand increased exponentially (200x) from about 10 million gallons in 2001 to about 2 billion gallons in 2016 (US Energy Information Administration, 2019). The feedstocks currently used for biodiesel production are plant-based, mostly vegetable oils from soybean, corn, sunflower, palm, and rapeseed (US Energy Information Administration, 2018). However, these could jeopardize food security by triggering competition over water, land, and fertilizer consumption.

Microalgae photosynthesize sunlight and carbon dioxide to energy-rich biomolecules, triacylglycerol (TAG), transesterifiable into biodiesel. In addition to biodiesel production, microalgae sequester greenhouse gases (Ono and Cuello, 2007; Packer, 2009), pivotal in circumventing climate change, are capable of bioremediating wastewaters (Markou and Georgakakis, 2011; Rawat et al., 2011), and synthesizing high-value pharmaceuticals and nutraceuticals as co-products (Raja et al., 2008; Shah et al., 2020). Several microalgae genera such as Phaeodactylum (Molina Grima et al., 1999), Nannochloropsis (Sukenik, 1999), Porphyridium (Su et al., 2016), Schizochytrium (Morita et al., 2006), and Chlorella (Levasseur et al., 2020), are cultivated heterotrophically for the production of high-value products. Microalgae offer significant advantages over yeast and bacteria, as they are photosynthetic and do not require any addition of organic sources of carbon, which is an essential criterion for establishing long-term sustainability for any industrial processes (Kazamia and Smith, 2014).

High production cost is the major caveat in the commercialization of microalgae-based biodiesel technology. Strain selection and improvement, lipid extraction to its fullest (Halim et al., 2012), identification and coextraction of valuable co-products (Song et al., 2015; Lin and Eudes, 2020), and synchronizing biomass and lipid accumulation (Rai et al., 2016) are the active areas of research. Biomass and lipid induction are inversely related and depend extensively on the growth conditions: optimal conditions support biomass while stress induces lipid accumulation. Stress conditions including light intensity (Mahong et al., 2012), temperature (Valledor et al., 2013; Abdelaziz et al., 2014), high $\mathrm{CO}_{2}$ (Lv et al., 2010), salinity (Mastrobuoni et al., 2012), oxidative stress (Wang et al., 2004), UV-irradiation (Wijffels and Barbosa, 2010), and nutrient limitation especially nitrogen (Longworth et al., 2012; Guarnieri et al., 2013), are associated with this phenotype. Prior knowledge and understanding of algal stress physiology, particularly carbon allocation between different biosynthetic pathways and regulatory mechanisms controlling the distribution of desired metabolites, is imperative to cut down the biodiesel production cost.

Availability of whole-genome sequences of several algae and associated technological advancements in analytical sciences and data analysis has facilitated multidimensional 'Algomics' analysis to pursue the microalgal system biology. Numerous transcriptomics and proteomics studies have been performed to investigate the stress-associated lipid accumulation in Chlamydomonas reinhardtii (Longworth et al., 2012; Blaby et al., 2013), Tisochrysis lutea (Garnier et al., 2014), Ostreococcus tauri (Martin et al., 2012), Haematococcus pluvialis (Recht et al., 2012), Nannochloropsis sp. (Dong et al., 2013; Karthikaichamy et al., 2017), Phaeodactylum tricornutum (Valenzuela et al., 2012; Yang et al., 2014), and Chlorella sp. (Cao et al., 2014; Rai et al., 2016 and 2017). However, limited metabolomic investigations are performed to understand the underlying molecular mechanisms involved in oil production from microalgae (Hirth et al., 2017; Shaikh et al., 2019).

Chlorella is one of the most widely studied microalgae and an industrial strain for biodiesel development (Ma et al., 2018). Chlorella is fastgrowing, accumulates $50-70 \%$ lipids (TAG)/g dry cell weight (DCW), could be a source of functional foods, nutraceuticals, food supplements, and is genetically manipulatable. Thus, chlorella biomass could be co-extracted for TAG and high-value pharmaceuticals and nutraceuticals to lower the biodiesel production cost. Here we report on a comprehensive metabolomics analysis of the Chlorella sp. FC2 IITG to get a holistic overview of the biochemical process involved in nitrogen-limitationinduced TAG and phytochemical accumulation. Data from the present study highlights the crosstalk between metabolic changes and stress physiology. High-value nutraceutical by-products are identified that could support the cut-down of biodiesel prices.

\section{Methods}

\subsection{Study design}

The objective of this work is to define the metabolomic analysis of the microalgae, Chlorella sp. FC2 IITG (here onwards referred to as FC2) due to nitrogen-limitation. Unbiased metabolomics analysis was performed for the FC2 during three nitrogen-limitation timepoints (40, 88, and $120 \mathrm{~h}$ ), representing different nitrogen-limitation severity (mild, moderate, and severe, respectively), based on the varied neutral lipid accumulation. Features are annotated by exact mass and MS/MS spectra corresponding to the spectral libraries publicly available, MoNA, METLIN, and GNPS. The data was also integrated with the proteomics findings reported previously by our group (Fig. 1). Microalgal culture conditions and physiological analysis are described previously (Rai et al., 2017).

\subsection{Strain selection, stress-physiology, and proteomics analysis}

The indigenous oleaginous green microalga FC2 was isolated by our research group from the North-Eastern part of India (Muthuraj et al., 2014). FC2 was cultured under a photoautotrophic condition in a $5.0 \mathrm{~L}$ automated bioreactor (Biostat B plus, Sartorius, Gettingen, Germany) containing 4.0 L of slightly modified BG11 medium following the previously described growth conditions (Rai et al., 2017). To classify the microalgae strain, 18S rRNA gene sequencing was performed. For optimal growth, we maintained nitrogen levels of $\sim 1.6 \mathrm{~g} / \mathrm{L}$, whereas nitrogen was removed entirely from media for nitrogen-limitation conditions. Synchronization of the yield and TAG accumulation in nitrogen-limited non-model FC2 was achieved by process engineering the $\mathrm{FC} 2$ cultivation through growing it in biphasic photoautotrophic conditions (first phase: optimal growth and second phase: nitrogen-limitation induced lipid accumulation). Neutral lipid accumulation was confirmed by Nile red staining following the previously described protocol (Rai et al., 2017). Furthermore, we performed unbiased globa quantitative protein profiling to unravel the underlying post-transcriptional mechanisms associated with the phenotype (Rai et al., 2017).

\subsection{Metabolite extraction}

Metabolites were extracted from four biological replicates of FC2 cells from each group: control $(0 \mathrm{~h})$, different nitrogen-limitation conditions: mild $(40 \mathrm{~h})$, moderate $(88 \mathrm{~h})$, and severe $(120 \mathrm{~h})$ (Fig. 1). Four hundred $\mu \mathrm{L}$ of chilled methanol: chloroform: water (4:3:1, Optima LC-MS grade Fisher Scientific) with $2 \mu \mathrm{M}$ caffeine (Analytical Standard, Sigma-Aldrich) was added to $100 \mathrm{mg}$ ground $\mathrm{FC} 2$ cells, vortexed for $30 \mathrm{~s}$, and incubated at $-80^{\circ} \mathrm{C}$ for $8 \mathrm{~h}$. The samples were centrifuged at $14,000 \times \mathrm{g}$ for $15 \mathrm{~min}$ at $4^{\circ} \mathrm{C}$ to pellet down the precipitated proteins. Polar metabolites were separated in the methanol and water phase, while non-polar metabolites were extracted in chloroform, non-polar solvent (Bligh and Dyer, 1959). Both polar and non-polar fractions were pooled together and evaporated to dryness in a vacuum concentrator. The dried extracts were then reconstituted in $50 \%$ acetonitrile and $0.1 \%$ formic acid $(100 \mu \mathrm{L})$, filtered through a 0.2 -micron syringe filter, and stored at $-80^{\circ} \mathrm{C}$ before liquid chromatography-mass spectrometry (LC-MS) analysis. 


\subsection{Metabolomics analysis and profiling}

A high-resolution LC-MS based comprehensive untargeted metabolomics profiling of FC2 extracts for four biological replicates from each group: control $(0 \mathrm{~h})$, different nitrogen-limitation conditions: mild $(40 \mathrm{~h})$, moderate $(88 \mathrm{~h})$, and severe (120 h) was performed (Fig. 1) and mobile phase B is $0.1 \%$ formic acid in $90 \%$ acetonitrile (LC-MS grade solvents, Fisher Scientific). Initial conditions were restored in $2 \mathrm{~min}$ ahead of 5 min of column re-equilibration. The HPLC system was coupled with an Agilent 6550 ifunnel Q-TOF LC-MS/MS system controlled by Agilent MassHunter ${ }^{\mathrm{TM}}$ software packages and equipped with an ESI source. MS1 spectra were acquired in positive electrospray ionization (+ESI) mode with

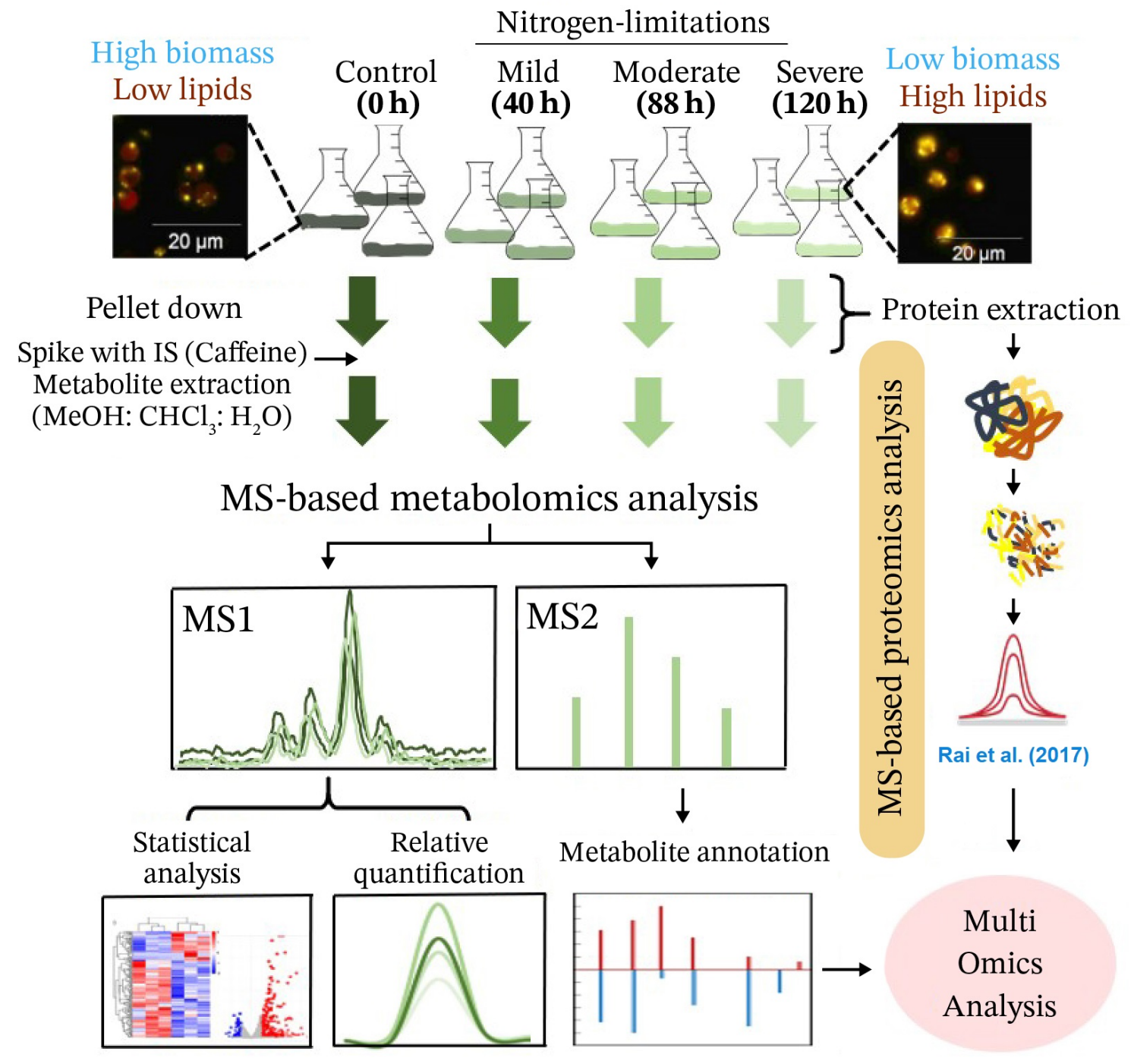

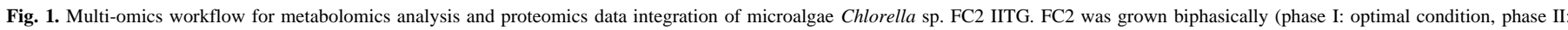

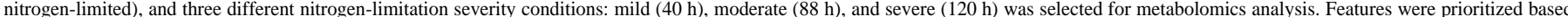

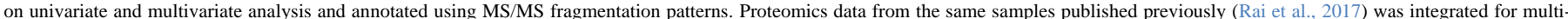
omics analysis to better understand metabolic tug-of-war between biomass and TAG assimilation during nitrogen-limitation.

\subsubsection{Instrumentation and data acquisitions}

MS and MS/MS analysis of the metabolites were performed as per our laboratory protocol (Patel et al., 2019). FC2 metabolite extracts $(6 \mu \mathrm{L})$ were chromatographically separated with an Agilent 1260 Infinity HPLC system with a Zorbax SB-AQ C18 column (100x2.1 mm) packed with $1.8 \mu \mathrm{m}$ particles (Agilent Technologies, Santa Clara, CA) using a gradient of 5\% mobile phase B increasing linearly to $80 \% \mathrm{~B}$ in $25 \mathrm{~min}$ at a flow rate of $0.3 \mathrm{~mL} / \mathrm{min}$, and temperature at $40^{\circ} \mathrm{C}$. The mobile phase $\mathrm{A}$ comprises $0.1 \%$ formic acid in water, a mass range of 50-1000 m/z. The instrument was externally calibrated, and mass spectra were recorded at $20 \mathrm{~K}$ resolution with ESI-L Low Concentration Tuning Mix (Agilent Technologies) daily. MS/MS analyses were conducted using nitrogen as collision gas and collision-induced dissociations (CID). Ten most abundant ions per MS1 scan were fragmented for data-dependent acquisition, and the spectra were collected. The samples were randomized to eliminate any biases, and a pooled quality control sample $(0,40,88$, and $120 \mathrm{~h})$ was run after every four samples to determine run-to-run variation. 


\subsubsection{Quality assessment of the data collection}

Agilent raw data files were converted to ".mzXML" files using MSconvert software (Holman et al., 2014). Raw data files were manually inspected in Agilent MassHunter ${ }^{\mathrm{TM}}$ software. The coefficient of variance (CV) for resuspension standard caffeine $\left([\mathrm{M}+\mathrm{H}]^{+}, \mathrm{m} / \mathrm{z}\right.$ 195.0882) was determined.

\subsubsection{Feature finding}

MS feature detection was performed using MZmine 2.53 in batch mode following the reported method (Gauglitz et al., 2020). The mzXML files were imported and cropped based on retention time (0-23 $\mathrm{min})$. The mass detection noise level for MS was set at 4.0E3 and 5.0E2 for MS/MS. Chromatograms were built with $\mathrm{ADAP}$ chromatogram builder, a min-height of 3.0E3, a min gr size in \# of scans of 3 , a gr intensity threshold of $9.0 \mathrm{E} 3$, an $\mathrm{m} / \mathrm{z}$ tolerance of $20.0 \mathrm{ppm}$. Chromatograms were deconvoluted using minimum local search (chromatographic limit: 60\%; search minimum in RT range (min): 0.05; minimum relative height: $15 \%$; min absolute height: $3.0 \mathrm{E} 3$, min ratio of peak top/edge: 1.5 and peak duration range ( $\mathrm{min}$ ) of $0.00-2.00$ ). Isotopic peaks were grouped with an $\mathrm{m} / \mathrm{z}$ tolerance of $20.0 \mathrm{ppm}$, a retention time tolerance of 0.1 , and a maximum charge of 3 . Features were aligned with $\mathrm{m} / \mathrm{z}$ tolerance of 20.0 $\mathrm{ppm}$, weight or $\mathrm{m} / \mathrm{z} 75 \%$, and weight for RT $25 \%$, with a retention time tolerance of $0.1 \mathrm{~min}$. Gap filling was done with an intensity tolerance of $10.0 \%$ and a retention time tolerance of $0.15 \mathrm{~min}$. Feature tables were filtered to include only features that contain a minimum of 8 peaks in a row. Duplicate peaks were filtered, and the feature table was exported.

\subsection{Data filtering, analysis, and visualization}

The data filtering was performed using MetaboAnalyst v4.0 (https://www.metaboanalyst.ca/). The data were filtered to create feature lists based on the number of entities detected in at least two conditions for the control $(0 \mathrm{~h})$ and nitrogen-limitation conditions $(40 \mathrm{~h}, 88 \mathrm{~h}$, and $120 \mathrm{~h})$. For a given condition of nitrogen-limitation, filtered entity lists were created based on the number of entities that passed $80 \%$ sample replicate thresholds. Four biological replicates were analyzed as one group after the ion intensity for each molecular ion was averaged across the replicates. Partial least squaresdiscriminant (PLS-DA) analysis and volcano plots were generated to visually demonstrate the variance of the metabolic phenotypes within the group on the sample files based on the variable: nitrogen-limitation. One-way ANOVA (p $<0.05)$ analysis resulted in lists of matrix table showing the comparison results for each pair of conditions as a p-value. Fold change $\geq 1.5$, and $p$-value $\leq 0.05$ was the criterion to select differentially expressed metabolites. Missing values were excluded from the calculations, and the results were compared. Features were annotated based on MS/MS spectra. All annotations are level 2 or 3, according to the 2007 metabolomics standards initiative (Sumner et al., 2007). A heat map (K-mean clustering) was generated for the metabolites.

\subsection{Statistical analyses}

One-way ANOVA was used to determine the statistical significance of the results. $p$-value $\leq 0.05$ was considered statistically significant.

\subsection{Data and software availability}

LC-MS/MS data from this paper have been deposited to massIVE with the dataset identifier MSV000085689 (ftp://massive.ucsd.edu/MSV000085689/).

\section{Results and Discussion}

\subsection{Nitrogen-limitation induces TAG accumulation in FC2 cells}

Unicellular microalgae are at the forefront of research efforts to develop technologies and model systems for renewable biodiesel production. Abiotic stresses strongly induce lipid accumulation in the form of TAG, convertible to biodiesel. A comprehensive understanding of the molecular mechanisms underlying the stress physiology to achieve the high yield and simultaneous accumulation of phytochemicals, including TAGs and polyunsaturated fatty acids (PUFA) is inevitable to strengthen the scope for microalgal biodiesel commercialization. Our group previously isolated the indigenous oleaginous green microalga FC2 from the North-Eastern part of India (Muthuraj et al. 2014). To classify the microalgae, $18 \mathrm{~S}$ rRNA gene of FC2 (Accession no.: JX154075.1) was sequenced, and as per the latest update in the 18S rRNA gene database, the strain could potentially be Chlorella sorokiniana. A phylogenetic tree was constructed with closely related species $(C$. vulgaris, C. pyrenoidosa, $C$. sorokiniana, $C$. singularis, etc.) and found that the strain FC2 falls close to $C$. sorokiniana as depicted in the phylogenetic tree (Fig S1). The novel microalgal strain "Chlorella sp. FC2 IITG" has been deposited to the National Collection of Industrial Microorganisms (NCIM) culture collection, National Chemical Laboratories (NCL), Pune, India, with a safe deposit number NCIM-5666.

Process engineering strategies employed in our previous study resulted in a maximum biomass titer of $17.7 \mathrm{~g} / \mathrm{L}$ and total lipid accumulation of 313 $\mathrm{mg} / \mathrm{L} . \mathrm{d}$, the highest reported to date among Chlorella sp. (Muthuraj et al., 2015). The lipid accumulation is higher than that reported for C. vulgaris grown in continuous nitrogen-limitation (Liu et al., 2016). Further, a $50.34 \%(\mathrm{w} / \mathrm{w}, \mathrm{DCW})$ higher neutral lipid storage in severe nitrogen-limited FC2 (120 h), as compared to $1 \%$ in optimal growth conditions, was reported (Fig. S2) (Rai et al., 2017). Also, nitrogen-limitation leads to timedependent down-regulation of protein, carbohydrate, and chlorophyl contents in FC2 (Fig. S2). The time points, i.e., three different nitrogenlimitation severity conditions: $40 \mathrm{~h}$ (mild), $88 \mathrm{~h}$ (moderate), and $120 \mathrm{~h}$ (severe), which represent the critical stages for neutral lipid accumulation, were selected for downstream 'Algomics' analysis. The major findings from the proteomics analysis are published previously (Rai et al., 2017). The present study highlights the metabolomics analysis and multi-omics data integration.

\subsection{Metabolome profile of nitrogen-limited FC2 cells}

Microalgal FC2 extracts were analyzed by a high-resolution Q-TOF mass spectrometer. The base peak MS chromatogram of all the conditions, control $(0 \mathrm{~h})$, and nitrogen-limitations (mild, moderate, and severe) were highly reproducible (Fig. 2a). A similar trend was noted with merely $14.7 \%$ coefficient of variance of caffeine (m/z 194.08), spiked as internal standard, for all biological replicates $(n=4)$ and experimental FC2 samples (control and nitrogen-limited; depicted in Figure 2a), which further confirms the reproducibility in sample processing and the LC-MS runs. PLS-DA clustered all the treatments and control conditions into four groups (Fig. $2 \mathrm{~b}$ ), based on significantly altered features (fold changes $\geq 1.5$, and $\mathrm{p}$-value $\leq 0.05)$. Nitrogen-limitation induced modulations of 430 significan features, of which 84 showed overlap in mild, moderate, and severe conditions. In contrast, 5, 52, and 113 features are expressed exclusively in mild, moderate, and severe nitrogen-limitation, respectively. Still, other features assist in the algal adaptation to more stringent nitrogen-limitation conditions (Fig. 2c). Similar patterns were found in the proteomics and metabolomics analysis, where the maximum number of proteins (57) (Rai et al., 2017) and features (132) were regulated during the transition from moderate to severe nitrogen-limitation (Fig. 2c), suggesting drastic metabolic re-organization to combat extreme nitrogen-limitation stress and enhance the chance of survival. Volcano plots showing p-values vs. $\log 2$ fold change of 40/0, 88/0, and 120/0 h are depicted in Figure 3a (list of significantly altered features are shown in Table S1). The data also shows a similar trend, i.e., a higher number of features are dysregulated in severe (339) nitrogen-limitations (Fig. 3a iii) followed by moderate (302) (Fig. 3a ii) and mild (133) (Fig. 3a i) stress conditions (Table S1).

\subsection{High-value molecules amass in nitrogen-limited FC2 cells}

Microalgae are promising third-generation biofuel candidates and future bio-based food/feedstocks with a unique lipid composition, high protein content, and a source of unlimited bioactive phytochemicals, referred to as "High-value molecules (HVMs)". Although produced in fewer amounts, these products have much higher economic value than whole, dried microalgal biomass. HVMs range from PUFA, pigments, proteins to carbohydrates, with diverse applications in food, feed, chemical, cosmetic, energy, and health industries (Eppink et al., 2019; Bhowmick et al., 2020) The content and compositions of HVMs are rooted in their genetic, biochemical, and physiological diversity, which provides 
a

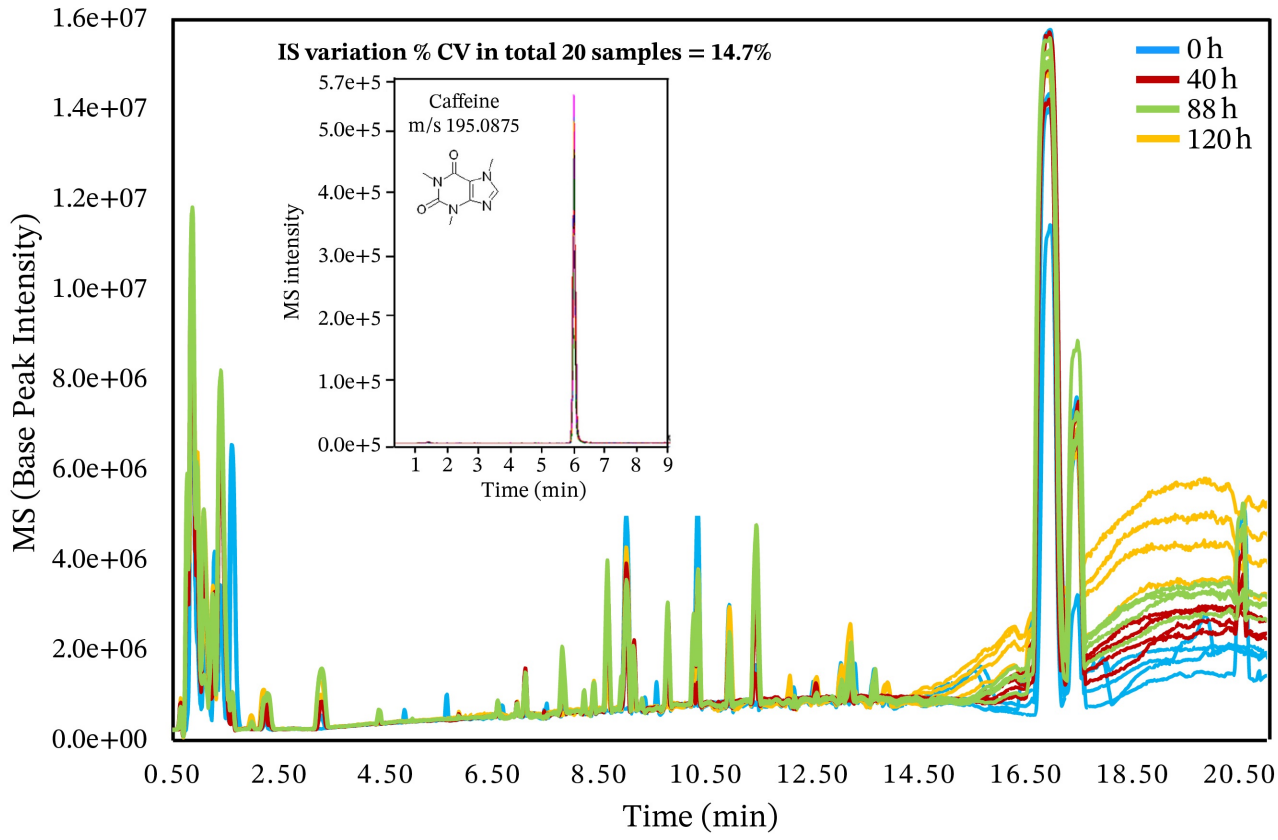

b
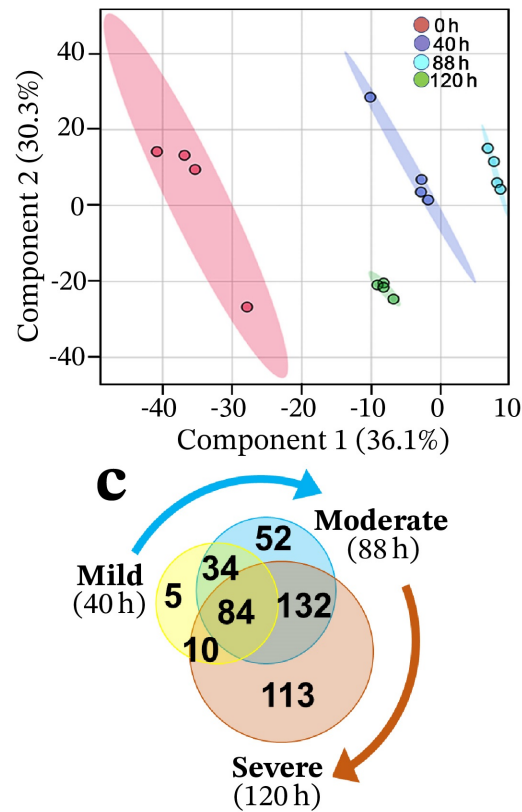

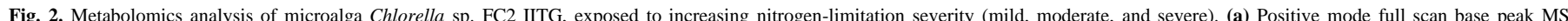

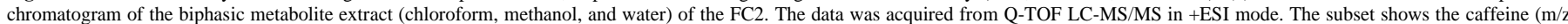

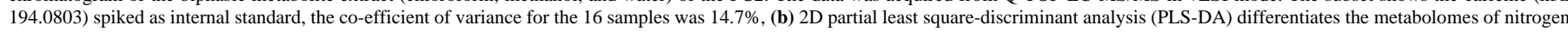

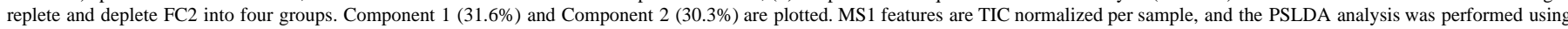
MetaboAnalyst, (c) Venn diagram showing the unique and overlapping differentially abundant features (one-way ANOVA, FDR $\leq 0.05$ ) in different nitrogen limitation severity. Consistently, more

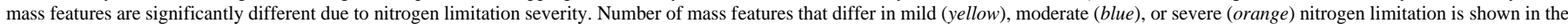
colored circles, with overlaps.

a

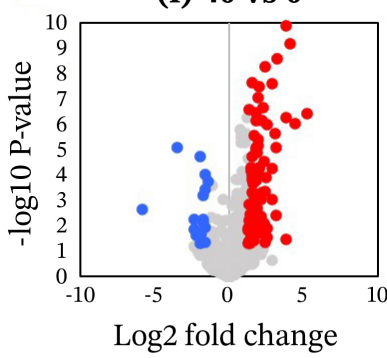

(ii) 88 vs 0

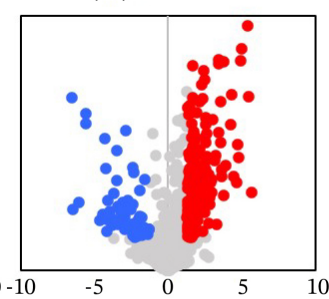

Log2 fold change (iii) 120 vs 0

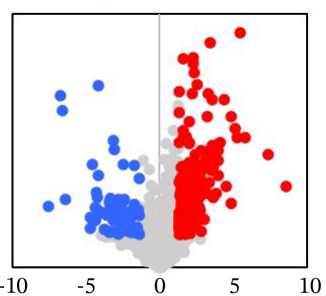

Log2 fold change
C (i) 9(S)-HpOTrE

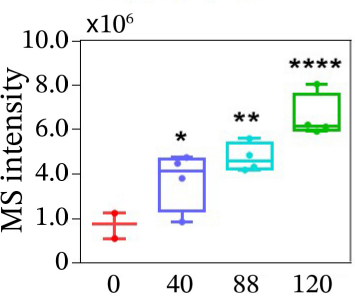

$\mathrm{N}$-limitation time $(\mathrm{h})$ (iii) TAG 59:10

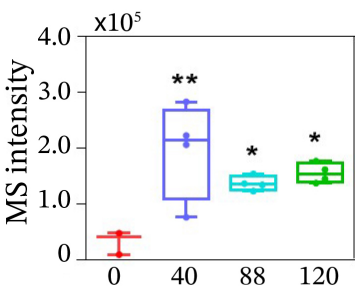

$\mathrm{N}$-limitation time(h)

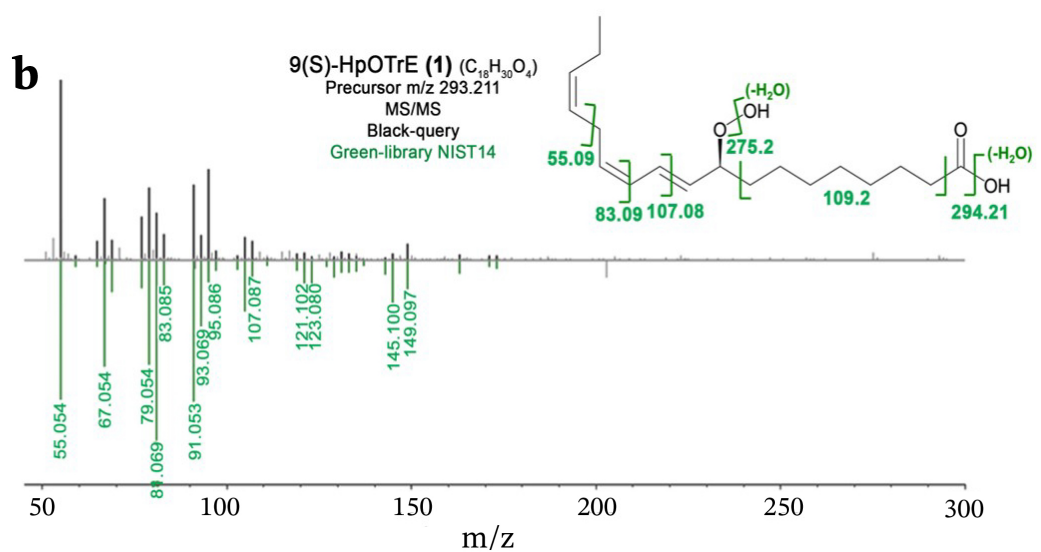

(ii) DGLA

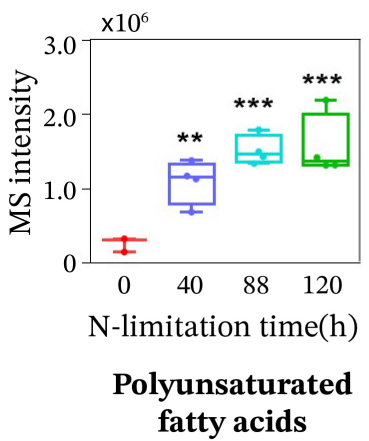

(iv) TAG 54:4

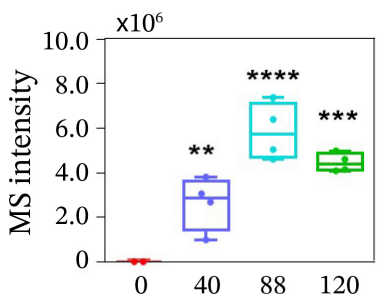

$\mathrm{N}$-limitation time(h)

Triacylglycerol accumulation

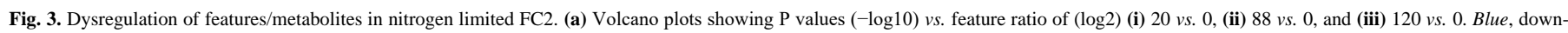

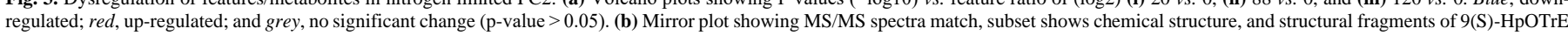

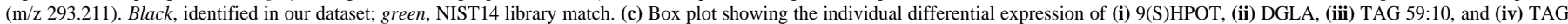
54:4. Results are shown as biological replicates and mean \pm SD. One-way ANOVA showed significant differences between groups. $(* \mathrm{P}<0.05, * * \mathrm{P}<0.01, * * * \mathrm{P}<0.001, * * * * \mathrm{P}<0.0001)$. 
opportunities to discover novel small molecules for industrial and health applications (Kim and Chojnacka, 2015). More than 4000 studies on microalgal phytochemicals were published between 1926 and 2016 (Michalak et al., 2017). Co-extraction of HVMs and TAG has immense potential to lower the microalgal biodiesel production costs, a major snag in the commercialization of this biofuel (Khoo et al., 2019). The first step towards isolating these bioactive molecules in a cost- and energy-efficient manner is their identification and quantitation. In the present study, we annotated ten significantly altered metabolites in the nitrogen-limited FC2 cells (detailed in Table 1) by mining online available MS/MS spectral libraries. The MS/MS spectral match of one such metabolite is shown in Figure 3b, and the subset shows the corresponding structural fragments. These ten metabolites are grouped into four biological classes: PUFAs, TAG, phytosterols, and terpenoids. We annotated two TAG species, namely, TAG 59:10 and TAG 54:4 (Table 1). Interestingly, a 200-fold higher storage of TAG 54:4 was noted (Fig. $3 \mathrm{c}$ iv) in moderate nitrogen-limitations ( $88 \mathrm{~h}$ ) compared to the optimal growth. Simultaneously, severe conditions led to the reduction in TAG 54:4, suggesting, scope to optimize nitrogen-limitation duration to sync FC2 yield and TAG induction. Also, we found a substantial accumulation of Dihydrotestosterone in moderate $(88 \mathrm{~h})$ and severe $(120 \mathrm{~h})$ nitrogen-limited FC2 cells (Table 1 and Fig. 4). Salinity stress-induced accumulation of phytosterols, ergosterol, and 7-dehydroporiferasterol, was reported in two different species of Dunaliella (Francavilla et al., 2010). Further, we found that nitrogen-limitation led to the accumulation of several diterpenoid and diterpene glycosides metabolites (listed in Table 1 and Fig. 4). However, terpenoids' extraction is technically challenging, with low or moderate yields (Moser and Pichler, 2019).

\subsection{Biorefining of TAGs and PUFAs could lower biodiesel production cost}

Microalgae are recognized as a rich source of PUFAs. Several PUFAs have penetrated the markets, while other classes of molecules, including polyphenols, phytosterols, terpenoids, and hormones, are still under active research. Fatty acids from microalgae have higher commercial importance than fish oils due to lower contaminations of methyl mercury, dioxins, and polychlorinated biphenyls (Ryckebosch et al., 2014). PUFAs from microalgae have an estimated market value of $140 \mathrm{USD} / \mathrm{kg}$ (Borowitzka 2013).

We found $4 \mathrm{x}$ and $6 \mathrm{x}$ higher accumulation of two different PUFA: 9(S)HpOTrE (Fig. 3c i) and dihomo-gamma-linolenic acid (Fig. 3c ii), respectively, in severe nitrogen-limitation conditions (120 h). 9(S)HpOTrE is a downstream product of alpha-linolenic acid (ALA), omega-3 fatty acid. The global omega-3 market size was valued at USD 2.49 billion in 2019 and is expected to expand at a compound annual growth rate of $7.7 \%$ over the forecast period (Grand View Research, 2020). Likewise, dihomogamma-linolenic acid (DGLA) is a precursor for several physiologically essential acids: arachidonic (ARA; C20:4n-6), eicosapentaenoic acid $(\mathrm{C} 20: 5 \mathrm{n}-3)$, and docosahexaenoic $(\mathrm{C} 22: 6 \mathrm{n}-3)$. The fact that FC2 accumulates PUFAs during nitrogen-limitation could be exploited to lower the biodiesel production cost by co-extracting with TAG. Industriallycompatible method for co-extracting phytochemicals was attempted (Delbrut et al., 2018; Santoro et al., 2019). Still, extensive research is needed to optimize the PUFA accumulation in stress-condition and extraction of phytochemicals to its fullest

Table 1.

List of differentially abundant metabolites identified in nitrogen-limited Chlorella sp. FC2 IITG

\begin{tabular}{|c|c|c|c|c|c|c|c|c|c|c|}
\hline \multirow{2}{*}{ No. } & \multirow{2}{*}{ Biological class } & \multirow{2}{*}{ Compounds } & \multirow{2}{*}{$\begin{array}{l}\text { Monoisotopic } \\
\text { mass }\end{array}$} & \multirow{2}{*}{$\mathbf{m} / \mathbf{z}$} & \multirow{2}{*}{ Adduct } & \multirow{2}{*}{$\begin{array}{c}\text { RT } \\
(\mathbf{m i n})\end{array}$} & \multicolumn{3}{|c|}{$\begin{array}{l}\text { Nitrogen-limitation } \\
\text { timepoints }\end{array}$} & \multirow{2}{*}{$\begin{array}{l}\text { Chemical } \\
\text { formula }\end{array}$} \\
\hline & & & & & & & $40 \mathrm{~h}$ & $88 \mathrm{~h}$ & $120 \mathrm{~h}$ & \\
\hline 1 & \multirow{2}{*}{$\begin{array}{l}\text { Polyunsaturated } \\
\text { fatty acids } \\
\text { (PUFA) }\end{array}$} & 9(S)HpOTrE & 310.2144 & 293.2091 & $\mathrm{M}+\mathrm{H}-\mathrm{H} 2 \mathrm{O}$ & 12.15 & 2.2 & 2.8 & 3.9 & $\mathrm{C} 18 \mathrm{H} 30 \mathrm{O} 4$ \\
\hline 2 & & Dihomo-gamma-linolenic acid (DGLA) & 306.2559 & 307.1493 & $\mathrm{M}+\mathrm{H}$ & 9.96 & 4.2 & 5.8 & 6.0 & $\mathrm{C} 20 \mathrm{H} 34 \mathrm{O} 2$ \\
\hline 3 & \multirow{3}{*}{ Triacylglycerides } & TAG 59:10 & 940.6581 & 963.4636 & $\mathrm{M}+\mathrm{Na}$ & 21.14 & 6.05 & 4.20 & 4.8 & C63H88O6 \\
\hline & & & & & & & & & & \\
\hline 4 & & TAG 54:4 & 882.7676 & 905.4246 & $\mathrm{M}+\mathrm{Na}$ & 15.61 & 100.9 & 224.3 & 170.7 & C57H102O6 \\
\hline 5 & Steroids & Dihydrotestosterone & 290.2246 & 291.1934 & $\mathrm{M}+\mathrm{H}$ & 11.63 & 2.8 & 4.3 & 4.6 & $\mathrm{C} 19 \mathrm{H} 30 \mathrm{O} 2$ \\
\hline 6 & \multirow{3}{*}{ Diterpenoid } & 14-Deoxyandrographolide & 334.2144 & 335.2159 & $\mathrm{M}+\mathrm{H}$ & 13.03 & 3.65 & 4.64 & 6.5 & $\mathrm{C} 20 \mathrm{H} 30 \mathrm{O} 4$ \\
\hline 7 & & 13-Labdene-2,8,15-triol & 324.2664 & 347.1791 & $\mathrm{M}+\mathrm{Na}$ & 11.54 & 4.2 & 6.3 & 9.2 & $\mathrm{C} 20 \mathrm{H} 36 \mathrm{O} 3$ \\
\hline 8 & & Annosquamosin C & 292.2402 & 293.2417 & $\mathrm{M}+\mathrm{H}$ & 19.43 & 7.5 & 2.8 & 7.9 & $\mathrm{C} 19 \mathrm{H} 32 \mathrm{O} 2$ \\
\hline 9 & \multirow{2}{*}{$\begin{array}{l}\text { Diterpene } \\
\text { glycosides }\end{array}$} & $\begin{array}{c}\text { (2R,3R,4S,5R,6R)-2-[[7-[(2R,3R,4R,5S)-3,4- } \\
\text { Dihydroxy-5-(hydroxymethyl)oxolan-2- } \\
\text { yl]oxy-2-ethenyl-2,4b,8,8-tetramethyl- } \\
\text { 4,4a,5,6,7,8a,9,10-octahydro-3H-phenanthren- } \\
\text { 3-yl]oxy]-6-methyloxane-3,4,5-triol }\end{array}$ & 582.3404 & 605.3279 & $\mathrm{M}+\mathrm{Na}$ & 11.45 & 5.84 & 9.51 & 10.91 & $\mathrm{C} 31 \mathrm{H} 50 \mathrm{O} 10$ \\
\hline 10 & & $\begin{array}{c}\text { c1-15(9-11-38-23(34)13-22(32)33)6-7-17- } \\
\text { 16(2)18(12-20-28(3,4)21(31)8-10- } \\
29(17,20) 5) 39-27-26(37) 25(36) 24(35) 19(14- \\
\text { 30)40-27/h9,17-21,24-27,30-31,35-37H,2,6- } \\
8,10-14 \mathrm{H} 2,1,3-5 \mathrm{H} 3,(\mathrm{H}, 32,33) / \mathrm{b} 15-9-\end{array}$ & 570.3040 & 571.3110 & $\mathrm{M}+\mathrm{H}$ & 10.15 & 2.76 & 3.82 & 7.26 & $\mathrm{C} 29 \mathrm{H} 46 \mathrm{O} 11$ \\
\hline
\end{tabular}




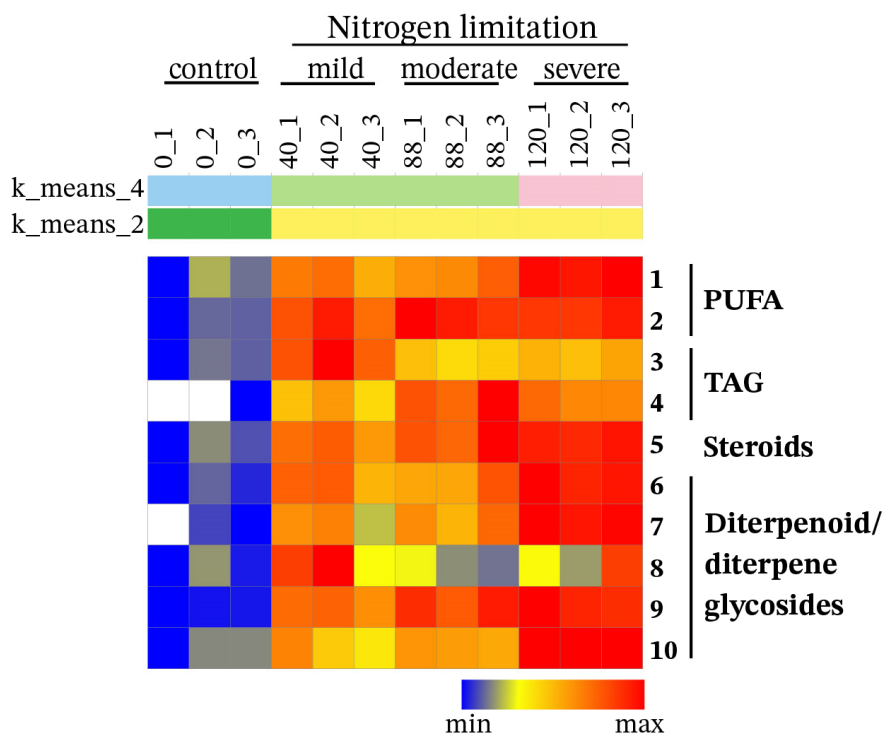

Fig. 4. Heat map illustrating the post-transcriptional dynamics of individual metabolites in response to nitrogen limitation (see details in Table 1). Blue, down-regulated; and red, upregulated.

\subsection{Nitrogen-limitation induces TAG synthesis in non-model microalgae FC2}

TAG is storage neutral lipids that have essential roles in energy homeostasis and carbon storage. TAG is considered a viable, sustainable source of biodiesel with commercial applications. Various abiotic stresses, including nitrogenlimitation, lead to TAG biosynthesis, which is a complex process. Systems biology studies revealed the readjustment of metabolism with a shift of carbon flux to TAG.

\subsubsection{Impaired carbon dioxide fixation}

Our physiological and proteomic analysis indicates that nitrogen-limitation reduces photosynthesis in FC2 cells. Considerable decline in the chlorophyll content and chlorophyll biosynthetic protein abundance, including porphobilinogen deaminase, was noted (Rai et al., 2017). Also, we found reduced regeneration of ribulose-1,5-bisphosphate carboxylase/oxygenase $(\mathrm{RuBis} \mathrm{CO})$ and significant downregulation of two of the rate-limiting enzymes phosphoribulokinase (PRK) and sedoheptulose-1,7-bisphosphatase (SBP), suggesting that carbon dioxide fixation is severely impaired in nitrogen-limited FC2 (Rai et al., 2017), which correlates well with the reduced overall yield. The nitrogen-limitation-induced decline in chlorophyll content is linked to rapid cessation in its synthesis and dilution by cellular growth rather than its degradation, as reported in C. reinhardtii (Juergens et al., 2015). We found upregulation in several enzymes, including peptidase, proteasomes, and ubiquitins, supporting the net degradation of protein and, therefore, the considerably decreased protein level (Rai et al., 2017). This would imply that the enormous carbon demand for TAG synthesis in nitrogen-limited FC 2 cells is met by catabolizing and reallocating carbon of proteins and membrane lipids. We also found overexpression of several of $\beta$-oxidation pathways enzymes that promote membrane lipids breakdown to provide both carbon and energy (Rai et al., 2017). The distribution of macromolecules such as carbohydrates, proteins, chlorophyll, and neutral lipids or TAG under different nitrogenlimitation ages $(0,40,88$, and $120 \mathrm{~h})$ is depicted in Figure S2. This shows a drastic increase in the neutral lipid content with a concomitant reduction in the protein, carbohydrate, and chlorophyll levels within the biomass, thus explaining the rearrangement of macromolecular compositions to form TAG. Besides, activity screening of enzymes such as ADP-gluco-pyro-phosphorylase (AGPase), acetyl-CoA carboxylase (ACCase), glycerol-3-phosphate dehydrogenase, and diacyl-glycerol transferase showed varied activity under nutrient-limitation and replete conditions in FC2 (Muthuraj et al., 2019). For instance, the activity of AGPase was reduced with a concomitant increase in
ACCase, suggesting the reduction of fluxes towards carbohydrate biosynthesis and associated redirection of carbon towards TAG biosynthesis under nitrogen limitation conditions (Muthuraj et al., 2019). Apart from their energy production role, these proteins, amino acids, and membrane lipids play an essential role as intermediates in replenishing the tricarboxylic acid (TCA) cycle for carbon recycling, and energy and reductant generation to meet the enhanced synthesis of fatty acids/TAG. Fatty acid biosynthesis is an expensive process; for instance, the biosynthesis of one unit of C16:0 requires eight molecules of NADPH and seven ATP molecules. Thus, nitrogen-limited FC2 must have robust biosynthesis pathways to fulfill the energy and reductant demand for oleagenicity. Simultaneously, the salvaged nitrogen is likely to be repartitioned to those most needed functions for maintaining cell viability against nitrogen-limitation and producing storage lipids (Park et al., 2015; Chen et al., 2017; Rai et al., 2017).

3.5.2. Activation of glycolysis, TCA cycle, and reductive pentose phosphate pathway ( $P P P P)$

All organisms are capable of synthesizing lipids, but only the oleaginous species accumulate significant quantities ( $>30 \% \mathrm{DCW})$, primarily due to the production of high levels of acetyl-CoA, the basic building block of fatty acids (Ratledge, 1994). Acetyl CoA is produced by oxidative decarboxylation of pyruvate, the end-product of glycolysis. We hypothesize that the pyruvate production is favored in nitrogen-limited FC2 by the significant up-regulation of several glycolysis pathway enzymes, namely, phosphoglycerate kinase (PGK), pyruvate kinase (PK), enolase (ENL), and triosephosphate isomerase (TPI) (Rai et al., 2017). Acetyl CoA also connects glycolysis and the citric acid cycle to yield citrate. It is possible that citrate is accumulated into the cytoplasm but could not be identified in our metabolomics study as we used a reverse-phase column for analysis. While the upregulation of isocitrate lyase in our proteomics study suggests the conversion of citrate to acetyl-CoA, thus providing substrates for de novo lipid biosynthesis (Fakas et al., 2009). Dynamic metabolic flux balance analysis conducted during the growth of the FC2 strain under both replete and nutrient limitation conditions also depicted an increase in the fluxes towards acetyl-CoA biosynthesis, which are further utilized by the organism for neutral lipid biosynthesis (Muthuraj et al. 2013). This redirection of fluxes to acetyl-CoA formation is characterized by increased fluxes in the glycolytic pathway during nutrient-limited photoautotrophic growth. The study showed a $57 \%$ increase in the flux towards acetyl-CoA biosynthesis from pyruvate and $63 \%$ reduction in flux towards citrate formation from acetyl-CoA under nutrient limitation conditions as compared to replete conditions (Muthuraj et al., 2013). Further enhanced phosphoenolpyruvate (PEP), pyruvate, and acetyl CoA led to the production of HVM (Fig. 5). Overall, nitrogen-limited FC2 cells maintained a flux to Pyruvate $\rightarrow$ Acetyl $\mathrm{CoA} \rightarrow$ fatty acids via enhancing glycolysis, TCA cycle, and reductive pentose phosphate pathway (rPPP).

\subsubsection{Fatty acid synthesis and TAG assembly}

We found that nitrogen-limitation enhances the expressions of several enzymes of fatty acid synthase (FAS) like acyl carrier proteins, hydroxy acyl-ACP dehydrogenase (HAD), and enoyl-ACP reductase (ENR) in FC2 (Rai et al., 2017). The up-regulation of these enzymes in a harmonized manner strongly stimulates the integration of acetyl-CoA into de novo synthesized fatty acids. Over 100 genes are identified to be involved in lipid metabolism in microalgae $C$. reinhardtii (Li-Beisson et al., 2015) and diatom P. tricornutum (Muhlroth et al., 2013) based on sequence analysis. Several genes involved in lipid metabolism are engineered to improve lipid accumulation in algae and higher plants; however, limited success is achieved to increase TAG accumulation (Iskandarov et al., 2016; Ajjawi et al., 2017), possibly due to the multifunctional roles of several of the enzymes. Surprisingly, overexpression of acetyl-CoA carboxylase (ACCase) in rapeseed has no significant change in seed lipid content, but a 5-fold higher lipid content in potatoes was reported (Klaus et al., 2004). Also, overexpression of 3-ketoacyl-acyl-carrier protein synthase III (KASIII) reduces the rate of lipid synthesis in Arabidopsis and rapeseed (Dehesh et al., 2001), which is consistent with our study, as no significan changes in KAS were seen in nitrogen-limited FC2. Also, using FAME 


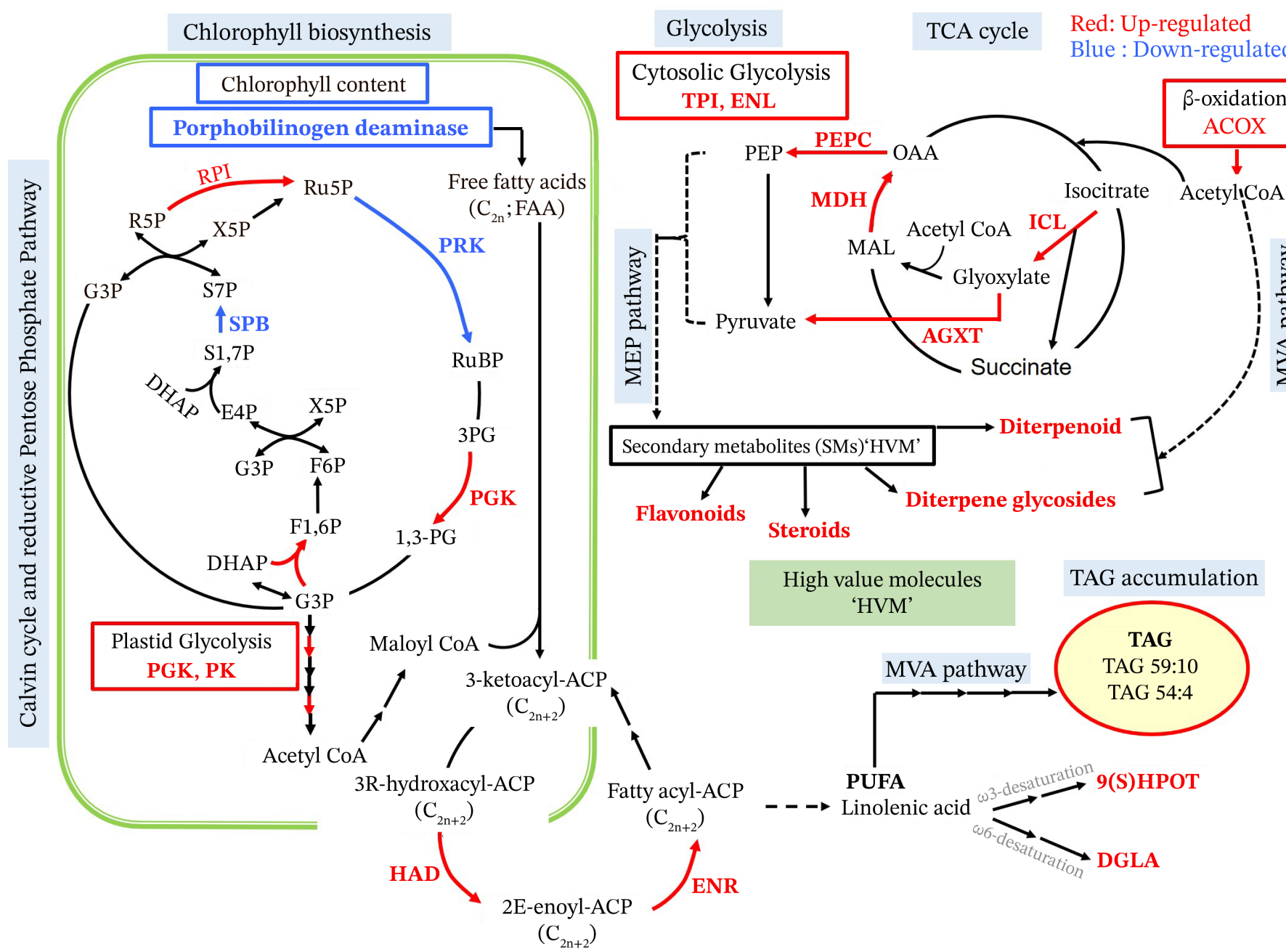

Fatty acid biosynthesis

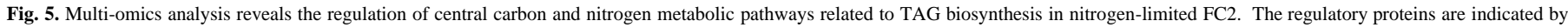
blue (down-regulated) and red (up-regulated) arrows, respectively. The metabolites accumulated during nitrogen limitation is shown in red.

profiling, we found a higher accumulation of C16:0 (palmitic acid), C18:1 (oleic acid), and C18:2 (linoleic acid) in nitrogen-limited FC2 (Muthuraj et al., 2014). C18:3 (LA) was $\sim 1-7 \%$ weight fraction of total fatty acids, which is less than the permissible limit of $12 \mathrm{~mol} \%$ as per European standards (Chisti, 2007). The de novo fatty acids are further desaturated to different levels by a series of desaturases, including omega- 6 fatty acid desaturase, and omega- 3 fatty acid desaturase. The ratio of unsaturated (monounsaturated plus polyunsaturated) to saturated fatty acid fractions in FC2 cells was $\sim 1.2$ (Muthuraj et al., 2014). We identified a significant up-regulation in two PUFAs, namely $9(\mathrm{~S}) \mathrm{HpOTrE}$ and DGLA in nitrogen-limited FC2 cells. Synergistic actions of several of the enzyme combinations, namely, GPDH, PGK, ENL, FBP, ALDO, TPI, and PK belonging to the glycolytic pathway, and RPI and RPE from rPPP provide cells with the flexibility to modulate energy levels, reducing power or a combination of these functions (Cantor and Sabatini, 2012). A similar pathway organization is reported in Scenedesmus dimorphus, Scenedesmus quadricauda (Sharma and Chauhan, 2016), and Mucor circinelloides (Tang et al., 2016), suggesting their strong role in redirecting the carbon-flux to TAG synthesis in nitrogenlimitations in oleaginous microalgae.

\section{Conclusions and future prospects}

Despite the extensive research on lipid production in microalgae, it is still challenging to pin-point precise molecular mechanism(s) driving nitrogen-limitation-induced TAG accumulation. This study focussed on the integrated analysis of non-omics (physiology) and omics (proteome and metabolome) of the non-model microalgae Chlorella sp. FC2 IITG metabolism (Fig. 5), cultivated in highly controlled parameter photobioreactors, under nitrogen-limitation conditions in a time-dependent manner. Metabolically, there is a tug-of-war ongoing between biomass and TAG assimilation during nitrogen-limitation. The reduced carbon flux limits TAG biosynthesis through central metabolism. Hence, overexpression of two of the rate-limiting enzymes PRK and SBP, under the control of the nitrogen-limitation induced promoter, could enhance $\mathrm{CO}_{2}$ fixation and enable better biomass and TAG assimilation.

From a commercialization point of view, more investigations are required to elucidate the target pathways, enzymes, and isoform(s) for increasing TAG productivity. In this regard, a more in-depth study of linolenic acid metabolic pathway enzymes, activity, and selectivity may 
help to unveil TAG formation and accumulation. Further, employing metabolomics, we annotated ten high-value molecules in the nitrogen-limited FC2, which could be co-extracted with TAG to lower the biodiesel production cost. In particular, nitrogen-limited FC2 cells accumulated 4-6x PUFAs. Our data suggest that FC2 is a promising microalgae strain for producing TAG and PUFAs simultaneously under moderate to severe nitrogen-limitation and could be an industrial strain of choice. Still, extensive research is needed to optimize the PUFA accumulation in stress-condition and extraction of phytochemicals to its fullest. A yet another attractive alternative from an environmental and technological point of view is to convert waste materials into fuels. The microalgae-based conversion of agro-industrial and municipal wastes into storage lipids could further lower biodiesel production costs and challenges for upcoming years.

\section{Acknowledgments}

We thank Manali Jadhav from the Centre for Research in Nanotechnology \& Science (CRNTS), Indian Institute of Technology Bombay, Mumbai, for performing LC/MS-MS runs. This research was supported by the DBT grant (BT/PR484/PBD/26/259/2011) and the DBT PAN IIT Centre for Bioenergy grant (BT/EB/PANIIT/2012) to SS and DD. VR was supported by DBT postdoctoral research associate fellowship. The IIT Bombay fellowship supported SKP.

\section{References}

[1] Abdelaziz, A.E.M., Ghosh, D., Hallenbeck, P.C., 2014. Characterization of growth and lipid production by Chlorella sp. PCH90, a microalga native to Quebec. Bioresour. Technol. 156, 20-28.

[2] Ajjawi, I., Verruto, J., Aqui, M., Soriaga, L.B., Coppersmith, J., Kwok, K., Peach, L., Orchard, E., Kalb, R., Xu, W., Carlson, T.J., Francis, K., Konigsfeld, K., Bartalis, J., Schultz, A., Lambert, W., Schwartz, A.S., Brown, R., Moellering, E.R., 2017. Lipid production in Nannochloropsis gaditana is doubled by decreasing expression of a single transcriptional regulator. Nat. Biotechnol. 35(7), 647-652.

[3] Bhowmick, S., Mazumdar, A., Moulick, A., Adam, V., 2020. Algal metabolites: an inevitable substitute for antibiotics. Biotechnol. Adv. 43, 107571

[4] Blaby, I.K., Glaesener, A.G., Mettler, T., Fitz-Gibbon, S.T., Gallaher, S.D., Liu, B., Boyle, N.R., Kropat, J., Stitt, M., Johnson, S., Benning, C., Pellegrini, M., Casero, D., Merchant, S.S., 2013. Systems-level analysis of nitrogen starvation-induced modifications of carbon metabolism in a Chlamydomonas reinhardtii starchless mutant. Plant Cell. 25(11), 43054323

[5] Bligh, E.G., Dyer, W.J., 1959. A rapid method of total lipid extraction and purification. Can. J. Biochem. Physiol. 37(8), 911-917.

[6] Borowitzka, M.A., 2013. High-value products from microalgae-their development and commercialization. J. Appl. Phycol. 25(3), 743-756.

[7] Cantor, J.R., Sabatini, D.M., 2012. Cancer cell metabolism: one hallmark, many faces. Cancer discovery. 2(10), 881-898.

[8] Cao, J., Yuan, H., Li, B., Yang, J., 2014. Significance evaluation of the effects of environmental factors on the lipid accumulation of Chlorella minutissima UTEX 2341 under low-nutrition heterotrophic condition. Bioresour. Technol. 152, 177-184.

[9] Chen, H., Zheng, Y., Zhan, J., He, C., Wang, Q., 2017. Comparative metabolic profiling of the lipid-producing green microalga Chlorella revealsthat nitrogen and carbon metabolic pathways contribute to lipid metabolism. Biotechnol. Biofuels. 10(1), 153.

[10] Chisti, Y., 2007. Biodiesel from microalgae. Biotechnol. Adv. 25(3), 294306.

[11] Colin, V.L., Rodriguez, A., Cristobal, H.A., 2011. The role of synthetic biology in the design of microbial cell factories for biofuel production. J. Biomed. Biotechnol. 2011, 601834.

[12] Dehesh, K., Tai, H., Edwards, P., Byrne, J., Jaworski, J.G., 2001. Overexpression of ${ }_{3}$-ketoacyl-acyl-carrier protein synthase IIIs in plants reduces the rate of lipid synthesis. Plant Physiol. 125(2), 1103-1114.

[13] Delbrut, A., Albina, P., Lapierre, T., Pradelles, R., Dubreucq, E., 2018. Fucoxanthin and polyunsaturated fatty acids co-extraction by a green process. Molecules. 23(4), 874
[14] Dong, H.P., Williams, E., Wang, D.Z., Xie, Z.X., Hsia, R.C., Jenck, A., Halden, R., Li, J., Chen, F., Place, A.R., 2013. Responses of Nannochloropsis oceanica IMET $_{1}$ to long-term nitrogen starvation and recovery. Plant Physiol. 162(2), 1110-1126.

[15] Eppink, M.H., Olivieri, G., Reith, H., van den Berg, C., Barbosa, M.J., Wijffels, R.H., 2019. From current algae products to future biorefinery practices: a review. Adv. Biochem. Eng. Biotechnol. 166, 99-123.

[16] Fakas, S., Papanikolaou, S., Galiotou-Panayotou, M., Komaitis, M., Aggelis, G., 2009. Biochemistry and biotechnology of single cell oil New Horiz. Biotechnol. 38-60.

[17] Francavilla, M., Trotta, P., Luque, R., 2010. Phytosterols from Dunaliella tertiolecta and Dunaliella salina: a potentially novel industrial application. Bioresour. Technol. 101(11), 4144-4150.

[18] Garnier, M., Carrier, G., Rogniaux, H., Nicolau, E., Bougaran, G. Saint-Jean, B., Cadoret, J.P., 2014. Comparative proteomics reveals proteins impacted by nitrogen deprivation in wild-type and high lipidaccumulating mutant strains of Tisochrysis lutea. J. Proteomics. 105, 107-120.

[19] Gauglitz, J.M., Aceves, C.M., Aksenov, A.A., Aleti, G., Almaliti, J. Bouslimani, A., Brown, E.A., Campeau, A., Caraballo-Rodriguez, A.M., Chaar, R., da Silva, R.R., Demko, A.M., Di Ottavio, F., Elijah, E., Ernst, M., Ferguson, L.P., Holmes, X., Jarmusch, A.K., Jiang, L., Kang, K.B., Koester, I., Kwan, B., Li, J., Li, Y., Melnik, A.V., Molina-Santiago, C., Ni, B., Oom, A.L., Panitchpakdi, M.W., Petras, D., Quinn, R., Sikora, N., Spengler, K., Teke, B., Tripathi, A., U1Hasan, S., van der Hooft, J.J.J., Vargas, F., Vrbanac, A., Vu, A.Q. Wang, S.C., Weldon, K., Wilson, K., Wozniak, J.M., Yoon, M., Bandeira, N., Dorrestein, P.C., 2020. Untargeted mass spectrometrybased metabolomics approach unveils molecular changes in raw and processed foods and beverages. Food Chem. 302, 125290.

[20] Grand View Research, 2020. Omega 3 market size, share \& trends analysis report by type (EPA, DHA, ALA), By source (marine source, plant source), by application, by region, and segment forecasts, 2020 2027. Res. markets. 100

[21] Guarnieri, M.T., Nag, A., Yang, S., Pienkos, P.T., 2013. Proteomic analysis of Chlorella vulgaris: potential targets for enhanced lipid accumulation. J. Proteomics 93, 245-253.

[22] Halim, R., Danquah, M.K., Webley, P.A., 2012. Extraction of oil from microalgae for biodiesel production: a review. Biotechnol. Adv. 30(3), 709-732.

[23] Hirth, M., Liverani, S., Mahlow, S., Bouget, F.Y., Pohnert, G., Sasso, S., 2017. Metabolic profiling identifies trehalose as an abundant and diurnally fluctuating metabolite in the microalga Ostreococcus tauri. Metabolomics. 13(6), 68.

[24] Holman, J.D., Tabb, D.L., Mallick, P., 2014. Employing proteowizard to convert raw mass spectrometry data. Curr. Protoc. Bioinf. 46(1), 13-24

[25] Iskandarov, U., Sitnik, S., Shtaida, N., Didi-Cohen, S., Leu, S. Khozin-Goldberg, I., Cohen, Z., Boussiba, S., 2016. Cloning and characterization of a GPAT-like gene from the microalga Lobosphaera incisa (Trebouxiophyceae): overexpression in Chlamydomonas reinhardtii enhances TAG production. J. Appl. Phycol. 28(2), 907-919.

[26] Juergens, M.T., Deshpande, R.R., Lucker, B.F., Park, J.J., Wang, H., Gargouri, M., Holguin, F.O., Disbrow, B., Schaub, T., Skepper, J.N. Kramer, D.M., Gang, D.R., Hicks, L.M., Shachar-Hill, Y., 2015. The regulation of photosynthetic structure and function during nitrogen deprivation in Chlamydomonas reinhardtii. Plant Physiol. 167(2), 558-573.

[27] Karthikaichamy, A., Deore, P., Rai, V., Bulach, D., Beardall, J., Noronha, S., Srivastava, S., 2017. Time for multiple extraction methods in proteomics? a comparison of three protein extraction methods in the eustigmatophyte alga Microchloropsis gaditana CCMP526. Omics: J. Integr. Biol. 21(11), 678-683.

[28] Kazamia, E., Smith, A.G., 2014. Assessing the environmental sustainability of biofuels. Trends Plant Sci. 19 (10), 615-618.

[29] Khoo, C.G., Dasan, Y.K., Lam, M.K., Lee, K.T., 2019. Algae biorefinery: review on a broad spectrum of downstream processes and products. Bioresour. Technol. 292, 121964. 
[30] Kim, S.K., Chojnacka, K., 2015. Marine algae extracts: processes, products, and applications. John Wiley \& Sons.

[31] Klaus, D., Ohlrogge, J.B., Neuhaus, H.E., Dormann, P., 2004. Increased fatty acid production in potato by engineering of acetyl-CoA carboxylase. Planta. 219(3), 389-396.

[32] Levasseur, W., Perre, P., Pozzobon, V., 2020. A review of high valueadded molecules production by microalgae in light of the classification. Biotechnol. Adv. 41, 107545.

[33] Li-Beisson, Y., Beisson, F., Riekhof, W., 2015. Metabolism of acyl-lipids in Chlamydomonas reinhardtii. Plant J. 82(3), 504-522.

[34] Lin, C.Y., Eudes, A., 2020. Strategies for the production of biochemicals in bioenergy crops. Biotechnol. Biofuels. 13, 1-25.

[35] Liu, T., Li, Y., Liu, F., Wang, C., 2016. The enhanced lipid accumulation in oleaginous microalga by the potential continuous nitrogen-limitation (CNL) strategy. Bioresour. Technol. 203, 150-159.

[36] Longworth, J., Noirel, J., Pandhal, J., Wright, P.C., Vaidyanathan, S., 2012. HILIC- and SCX-based quantitative proteomics of Chlamydomonas reinhardtii during nitrogen starvation induced lipid and carbohydrate accumulation. J. Proteome Res. 11(12), 5959-5971.

[37] Lv, J.M., Cheng, L.H., Xu, X.H., Zhang, L., Chen, H.L., 2010. Enhanced lipid production of Chlorella vulgaris by adjustment of cultivation conditions. Bioresour. Technol. 101(17), 6797-6804.

[38] Ma, X., Gao, M., Gao, Z., Wang, J., Zhang, M., Ma, Y., Wang, Q., 2018. Past, current, and future research on microalga-derived biodiesel: a critical review and bibliometric analysis. Environ. Sci. Pollut. Res. Int. 25(11), 10596-10610.

[39] Mahong, B., Roytrakul, S., Phaonaklop, N., Wongratana, J., Yokthongwattana, K., 2012. Proteomic analysis of a model unicellular green alga, Chlamydomonas reinhardtii, during short-term exposure to irradiance stress reveals significant down regulation of several heat-shock proteins. Planta. 235(3), 499-511.

[40] Markou, G., Georgakakis, D., 2011. Cultivation of filamentous cyanobacteria (blue-green algae) in agro-industrial wastes and wastewaters: a review. Appl. Energy. 88(10), 3389-3401.

[41] Martin, S.F., Munagapati, V.S., Salvo-Chirnside, E., Kerr, L.E., Le Bihan, T., 2012. Proteome turnover in the green alga Ostreococcus tauri by time course ${ }^{15} \mathrm{~N}$ metabolic labeling mass spectrometry. J. Proteome Res. 11(1), 476-486.

[42] Mastrobuoni, G., Irgang, S., Pietzke, M., Assmus, H.E., Wenzel, M., Schulze, W.X., Kempa, S., 2012. Proteome dynamics and early salt stress response of the photosynthetic organism Chlamydomonas reinhardtii. BMC Genomics. 13(1), 1-13.

[43] Michalak, I., Chojnacka, K., Saeid, A., 2017. Plant Growth Biostimulants, Dietary Feed Supplements and Cosmetics Formulated with Supercritical $\mathrm{CO}_{2}$ Algal Extracts. Molecules. 22(1), 66.

[44] Molina Grima, E., Garcia Camacho, F., Ácien Fernandez, F.G., 1999. Production of EPA from Phaeodactylum tricornutum, in: Cohen, Z. (Ed.), chemicals from microalgae. Taylor \& Francis, London, UK, pp. 57-92.

[45] Morita, E., Kumon, Y., Nakahara, T., Kagiwada, S., Noguchi, T., 2006. Docosahexaenoic acid production and lipid-body formation in Schizochytrium limacinum SR21. Mar. Biotechnol. (NY). 8(3), 319-327.

[46] Moser, S., Pichler, H., 2019. Identifying and engineering the ideal microbial terpenoid production host. Appl. Microbiol. Biotechnol. 103(14), 5501-5516.

[47] Muhlroth, A., Li, K., Rokke, G., Winge, P., Olsen, Y., HohmannMarriott, M.F., Vadstein, O., Bones, A.M., 2013. Pathways of lipid metabolism in marine algae, co-expression network, bottlenecks and candidate genes for enhanced production of EPA and DHA in species of Chromista. Mar. Drugs. 11(11), 4662-4697.

[48] Muthuraj, M., Kumar, V., Palabhanvi, B., Das, D., 2014. Evaluation of indigenous microalgal isolate Chlorella sp. FC2 IITG as a cell factory for biodiesel production and scale up in outdoor conditions. J. Ind. Microbial. Biotechnol. 41(3), 499-511.

[49] Muthuraj, M., Kumar, V., Palabhanvi, B., Das, D., 2015. Process engineering for photoautotrophic cultivation of high cell density lipid rich biomass of Chlorella sp. FC2 IITG. Bioenergy Res. 8(2), 726-739.

[50] Muthuraj, M., Palabhanvi, B., Misra, S., Kumar, V., Sivalingavasu, K., Das, D., 2013. Flux balance analysis of Chlorella sp. FC 2 IITG under photoautotrophic and heterotrophic growth conditions. Photosynth. Res. $118(1), 167-179$.
[51] Muthuraj, M., Selvaraj, B., Palabhanvi, B., Kumar, V., Das, D., 2019 Enhanced lipid content in Chlorella sp. $\mathrm{FC}_{2}$ IITG via high energy irradiation mutagenesis. Korean J. Chem. Eng. 36(1), 63-70.

[52] Ono, E., Cuello, J.L., 2007. Carbon dioxide mitigation using thermophilic cyanobacteria. Biosyst. Eng. 96(1), 129-134.

[53] Packer, M., 2009. Algal capture of carbon dioxide: biomass generation as a tool for greenhouse gas mitigation with reference to New Zealand energy strategy and policy. Energy Policy. 37(9), 34283437

[54] Park, J.J., Wang, H., Gargouri, M., Deshpande, R.R., Skepper, J.N. Holguin, F.O., Juergens, M.T., Shachar-Hill, Y., Hicks, L.M., Gang, D.R., 2015. The response of Chlamydomonas reinhardtii to nitrogen deprivation: a systems biology analysis. Plant J. 81(4), 611-624.

[55] Patel, S.K., Rajora, N., Kumar, S., Sahu, A., Kochar, S.K., Krishna, C.M., Srivastava, S., 2019. Rapid discrimination of malaria- anc dengue-infected patients sera using raman spectroscopy. Anal. Chem. 91(11), 7054-7062.

[56] Rai, V., Karthikaichamy, A., Das, D., Noronha, S., Wangikar, P.P. Srivastava, S., 2016. Multi-omics frontiers in algal research: techniques and progress to explore biofuels in the postgenomics world. OMICS. 20(7), 387-399.

[57] Rai, V., Muthuraj, M., Gandhi, M.N., Das, D., Srivastava, S., 2017. Real-time iTRAQ-based proteome profiling revealed the central metabolism involved in nitrogen starvation induced lipid accumulation in microalgae. Sci. Rep. 7(1), 1-16.

[58] Raja, R., Hemaiswarya, S., Kumar, N.A., Sridhar, S., Rengasamy, R. 2008. A perspective on the biotechnological potential of microalgae Crit. Rev. Microbiol. 34(2), 77-88.

[59] Ratledge, C., 1994. Yeasts, Moulds, Algae and Bacteria as Sources of Lipids. Technol. Adv in Improved Altern. Source Lipids. Springer, Boston. 235-291.

[60] Rawat, I., Kumar, R., Mutanda, T., Bux, F., 2011. Dual role of microalgae: phycoremediation of domestic wastewater and biomass production for sustainable biofuels production. Appl. Energy. 88(10), 3411-3424.

[61] Recht, L., Zarka, A., Boussiba, S., 2012. Patterns of carbohydrate and fatty acid changes under nitrogen starvation in the microalgae Haematococcus pluvialis and Nannochloropsis sp. Appl. Microbiol. Biotechnol. 94(6), 1495-1503.

[62] Ryckebosch, E., Bruneel, C., Termote-Verhalle, R., Goiris, K. Muylaert, K., Foubert, I., 2014. Nutritional evaluation of microalgae oils rich in omega-3 long chain polyunsaturated fatty acids as an alternative for fish oil. Food Chem. 160, 393-400.

[63] Santoro, I., Nardi, M., Benincasa, C., Costanzo, P., Giordano, G., Procopio, A., Sindona, G., 2019. Sustainable and selective extraction of lipids and bioactive compounds from microalgae. Molecules. 24(23), 4347.

[64] Shah, S.A.A., Hassan, S.S.U., Bungau, S., Si, Y., Xu, H., Rahman, M.H., Behl, T., Gitea, D., Pavel, F.M., Corb Aron, R.A., Pasca, B. Nemeth, S., 2020. Chemically diverse and biologically active secondary metabolites from marine Phylum chlorophyta. Mar. Drugs. 18(10), 493

[65] Shaikh, K.M., Nesamma, A.A., Abdin, M.Z., Jutur, P.P., 2019 Molecular profiling of an oleaginous trebouxiophycean alga Parachlorella kessleri subjected to nutrient deprivation for enhanced biofuel production. Biotechnol. Biofuels. 12(1), 1-15.

[66] Sharma, T., Chauhan, R.S., 2016. Comparative transcriptomics reveals molecular components associated with differential lipic accumulation between microalgal sp., Scenedesmus dimorphus and Scenedesmus quadricauda. Algal Res. 19, 109-122.

[67] Song, X., Zang, X., Zhang, X., 2015. Production of high docosahexaenoic acid by Schizochytrium sp. using low-cost raw materials from food industry. J. Oleo Sci. 64, 14164.

[68] Su, G., Jiao, K., Chang, J., Li, Z., Guo, X., Sun, Y., Zeng, X., Lu, Y., Lin, L., 2016. Enhancing total fatty acids and arachidonic acid production by the red microalgae Porphyridium purpureum. Bioresour. Bioprocess. 3(1), 33.

[69] Sukenik, A., 1999. Production of eicosapentaenoic acid by the marine eustigmatophyte Nannochloropsis, in: Cohen, Z. (Ed.), Chemicals from Microalgae. Taylor \& Francis, London, UK, pp. 41-56. 
[70] Sumner, L.W., Amberg, A., Barrett, D., Beale, M.H., Beger, R., Daykin, C.A., Fan, T.W., Fiehn, O., Goodacre, R., Griffin, J.L., Hankemeier, T., Hardy, N., Harnly, J., Higashi, R., Kopka, J., Lane, A.N., Lindon, J.C., Marriott, P., Nicholls, A.W., Reily, M.D., Thaden, J.J., Viant, M.R., 2007. Proposed minimum reporting standards for chemical analysis Chemical Analysis Working Group (CAWG) Metabolomics Standards Initiative (MSI). Metabolomics. 3(3), 211-221

[71] Tang, X., Zan, X., Zhao, L., Chen, H., Chen, Y.Q., Chen, W., Song, Y., Ratledge, C., 2016. Proteomics analysis of high lipid-producing strain Mucor circinelloides WJ11: an explanation for the mechanism of lipid accumulation at the proteomic level. Microb. Cell Fact. 15(1), 35

[72] US Energy Information Administration, March 2018.

[73] US Energy Information Administration, May 2019.

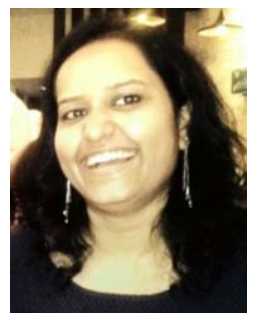

Dr. Vineeta Rai is a postdoctoral fellow in North Carolina State University, NC, USA. She received $\mathrm{Ph} . \mathrm{D}$. in Life sciences from DBT-Institute of Life sciences/Utkal University, Bhubaneswar, India. She serves as an editorial board member for the Indian Journal of Genetics and Molecular Research and topical editor for Plants. Her research interest includes multi-omics analysis, sustainable bioprocess for food and biofuel production, and value-added biochemicals synthesis.

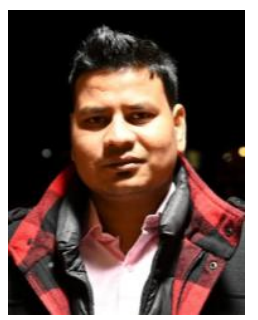

Dr. Sandip Kumar Patel is a postdoctoral fellow in Buck Institute for Research on Aging, CA, USA. He received Ph.D. from the Indian Institute of Technology Bombay, Mumbai, India. His research interests are to identify biomarkers using mass spectrometry-based proteomics and metabolomics.

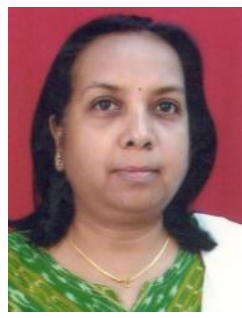

Dr. Mayuri Gandhi is a scientist at at Indian Institute of Technology Bombay, India. She received Ph.D. in Analytical Chemistry from Mumbai University. Her research interest include synthesis \& charecterisation of Quantum dots (QDs), NIR nanophosphors, upconverting nanophosphors, mesoporous hydroxyapetite, scintillators \& multimodal nanomaterials for different applications such as labels for protein, optical imaging, drug delivery, biosensors, nuclear scintilltors, quantum cutting material for solar cells etc., mass spectrometry for quantification \& biomarkers for protein from cancer.
[74] Valenzuela, J., Mazurie, A., Carlson, R.P., Gerlach, R., Cooksey, K.E., Peyton, B.M., Fields, M.W., 2012. Potential role of multiple carbon fixation pathways during lipid accumulation in Phaeodactylum tricornutum. Biotechnol. Biofuels. 5(1), 40.

[75] Valledor, L., Furuhashi, T., Hanak, A.M., Weckwerth, W., 2013. Systemic cold stress adaptation of Chlamydomonas reinhardtii. Mol. Cell. Proteomics. 12(8), 2032-2047.

[76] Wang, S.B., Chen, F., Sommerfeld, M., Hu, Q., 2004. Proteomic analysis of molecular response to oxidative stress by the green alga Haematococcus pluvialis (Chlorophyceae). Planta. 220(1), 17-29.

[77] Wijffels, R.H., Barbosa, M.J., 2010. An outlook on microalgal biofuels. Science. 329(5993), 796-799.

[78] Yang, Z.K., Ma, Y.H., Zheng, J.W., Yang, W.D., Liu, J.S., Li, H.Y., 2014. Proteomics to reveal metabolic network shifts towards lipid accumulation following nitrogen deprivation in the diatom Phaeodactylum tricornutum. J. Appl. Phycol. 26(1), 73-82.

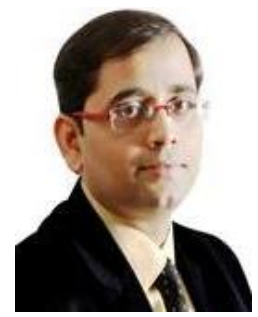

Dr. Sanjeeva Srivastava is a professor at Indian Institute of Technology Bombay, India. He received his $\mathrm{Ph} . \mathrm{D}$. from University of Alberta, Canada. He serves as a Deputy Editor-in-chief for Current Pharmacogenomics and Personalized Medicine and associate editor for Journal of Integrated OMICS, The International Journal of Biotechnology Applications, The International Journal of Drug Discovery, International Journal of Proteins and Peptides and International Journal of Systems Biology. His research interest is to employ high throughput proteomic techniques such as two-dimensional difference in gel electrophoresis, mass spectrometry and protein microarray etc. for biomarker discovery in cancer \& tropical diseases of India such as malaria, study protein-protein interactions and drug target discovery.

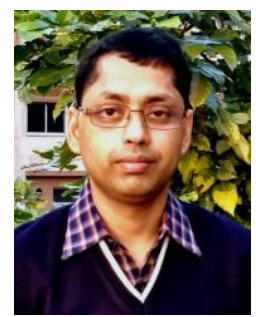

Dr. Debasish Das is a professor at Indian Institute of Technology Guwahati, India. He received his $\mathrm{Ph} . \mathrm{D}$. from Indian Institute of Technology Bombay, India. His research interests includes microalgae biofuel production, microalgal biotechnology, development of Clostridium sp. as a cell factory for butanol production, and sustainable bioprocess for synthesis of valueadded biochemicals.

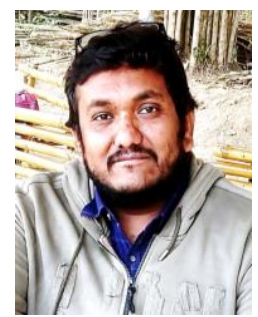

Dr. Muthusivarampandian Muthuraj is currently engaged as an Assistant Professor at the Department of Bioengineering, National Institute of Technology Agartala, India. He received his $\mathrm{Ph} . \mathrm{D}$. from the Indian Institute of Technology Guwahati, India. His research interests include bioenergy production, microalgal biotechnology, and sustainable bioprocess for synthesis of valueadded biochemicals. 
Supplementary data

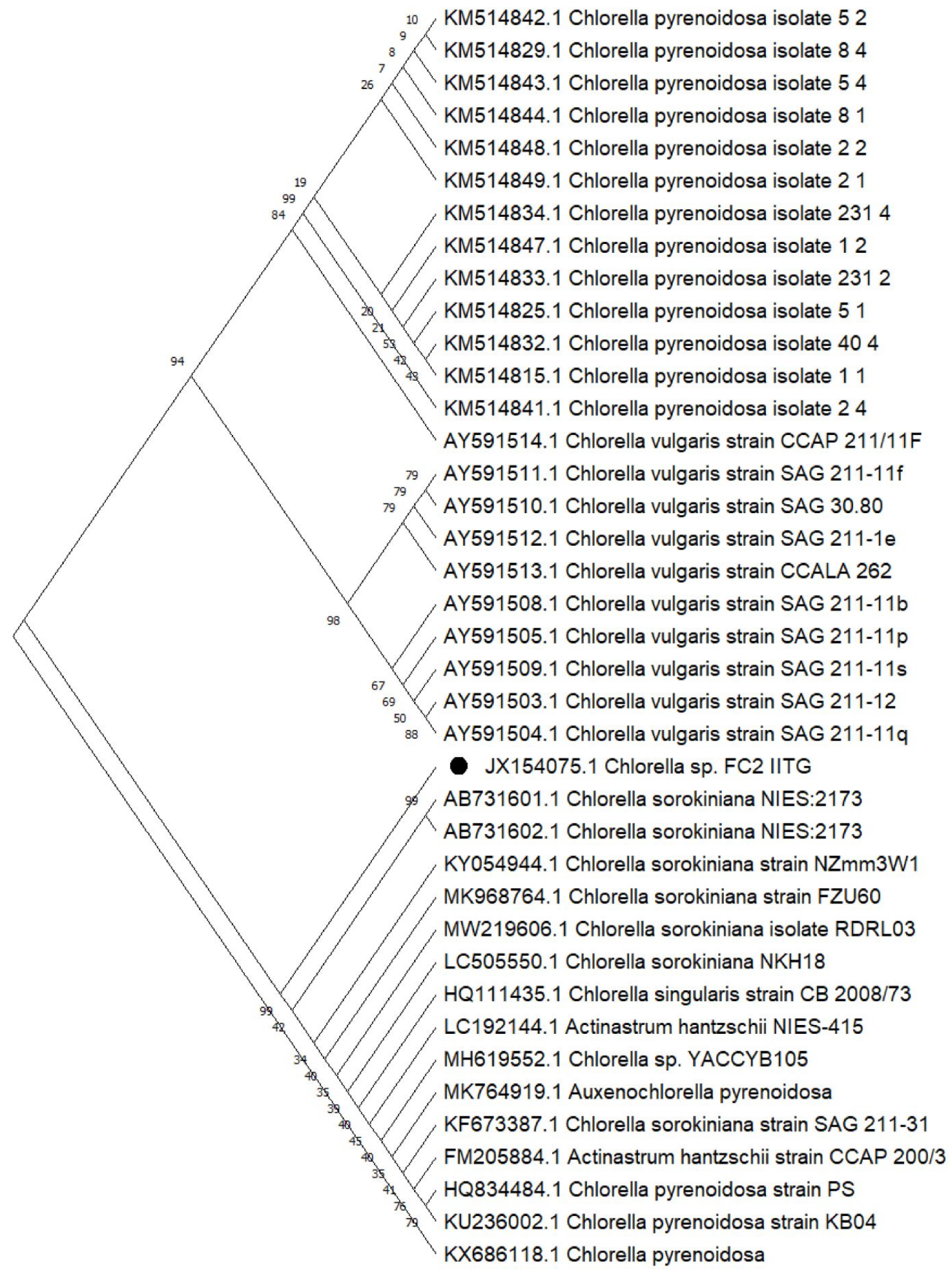

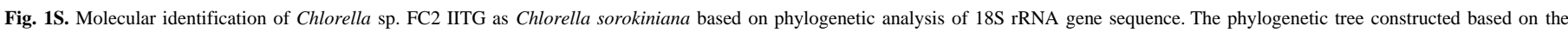
neighbour joining method with bootstrap analysis $(1,000$ replicates in \%) is shown. The strain used in the present study is marked with a filled circle. 


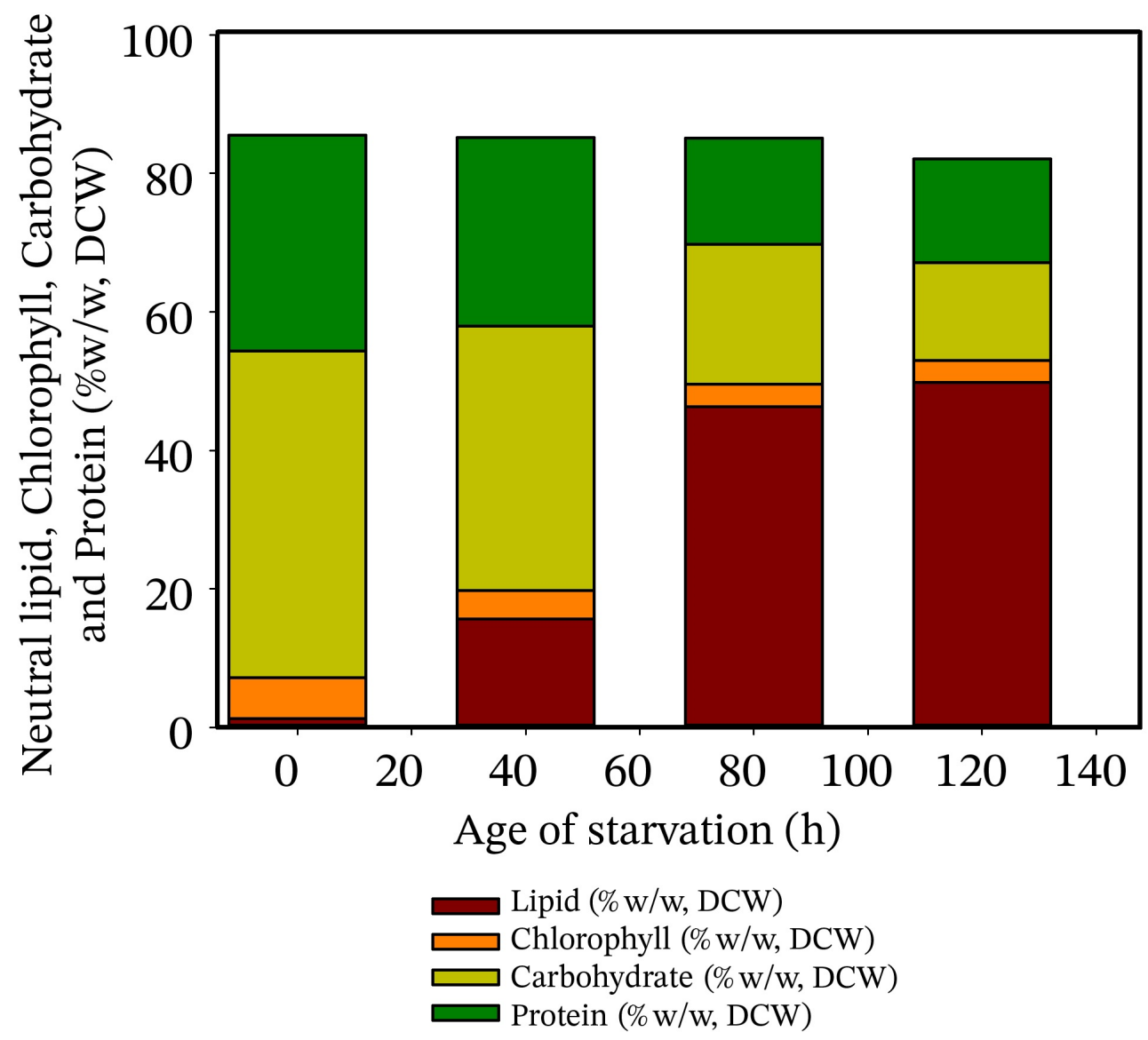

Fig. 2S. Adjustments in macromolecular compositions of microalgal biomass grown under optimal condition $(0 \mathrm{~h})$ and nitrogen-limitation conditions $(44,80$, and $120 \mathrm{~h})$ in $\% \mathrm{w} / \mathrm{w}, \mathrm{DCW}$.

Table S1.

Complete list of significantly altered features in mild $(40 \mathrm{~h})$, moderate $(88 \mathrm{~h})$, and severe $(120 \mathrm{~h})$ nitrogen-limitation as compared to optimal growth $(0 \mathrm{~h})$ in Chlorella sp. FC2 IITG. https://www.biofueljournal.com/jufile?ar_sfile=1250325 